\title{
Antibacterial Use of Macroalgae Compounds against Foodborne Pathogens
}

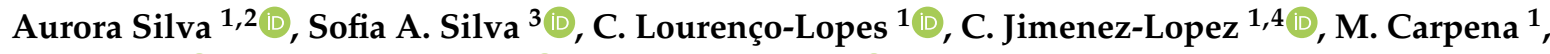 \\ P. Gullón ${ }^{1} \mathbb{D}$, M. Fraga-Corral 1,4 ${ }^{\mathbb{D}}$, V. F. Domingues ${ }^{2} \mathbb{D}$, M. Fátima Barroso ${ }^{2}$, \\ J. Simal-Gandara ${ }^{1, *(\mathbb{D}) \text { and M. A. Prieto }}{ }^{1, * \mathbb{D}}$
}

1 Nutrition and Bromatology Group, Department of Analytical and Food Chemistry, Faculty of Food Science and Technology, Ourense Campus, University of Vigo, E32004 Ourense, Spain; mass@isep.ipp.pt (A.S.); c.lopes@uvigo.es (C.L.-L.); cecilia.jimenez.lopez@uvigo.es (C.J.-L.);

maria.carpena.rodriguez@uvigo.es (M.C.); patrigullon@gmail.com (P.G.); mfraga@uvigo.es (M.F.-C.)

2 REQUIMTE/LAQV, Instituto Superior de Engenharia do Porto, Instituto Politécnico do Porto, Rua Dr António Bernardino de Almeida 431, 4200-072 Porto, Portugal; vfd@isep.ipp.pt (V.F.D.); MFSBA@isep.ipp.pt (M.F.B.)

3 Departamento de Química, Universidade de Aveiro, 3810-168 Aveiro, Portugal; sofia.silva96@gmail.com

4 Centro de Investigação de Montanha (CIMO), Instituto Politécnico de Bragança, Campus de Santa Apolonia, 5300-253 Bragança, Portugal

* Correspondence: jsimal@uvigo.es (J.S.-G.); mprieto@uvigo.es (M.A.P.)

Received: 19 August 2020; Accepted: 13 October 2020; Published: 17 October 2020

check for updates

\begin{abstract}
The search for food resources is a constant in human history. Nowadays, the search for natural and safe food supplies is of foremost importance. Accordingly, there is a renewed interest in eco-friendly and natural products for substitution of synthetic additives. In addition, microbial contamination of food products during their obtaining and distribution processes is still a sanitary issue, and an important target for the food industry is to avoid food contamination and its related foodborne illnesses. These diseases are fundamentally caused by certain microorganisms listed in this review and classified according to their Gram negative or positive character. Algae have proven to possess high nutritional value and a wide variety of biological properties due to their content in active compounds. Among these capabilities, macroalgae are recognized for having antimicrobial properties. Thus, the present paper revises the actual knowledge of microbial contaminants in the food industry and proposes antimicrobial algal compounds against those pathogenic bacteria responsible for food contamination as valuable molecules for its growth inhibition. The capacity of algae extracts to inhibit some major food pathogen growth was assessed. Moreover, the main applications of these compounds in the food industry were discussed while considering their favorable effects in terms of food safety and quality control.
\end{abstract}

Keywords: macroalgae; microbial contaminants; antimicrobial compounds; food industry; safety and quality

\section{General View on Algae Compounds}

Algae encompass a wide variety of photosynthetic living beings, usually aquatic, that comprise thousands of species. Even though the taxonomic classification of algae is a delicate subject, since each researcher classifies them according to different criteria, generally, marine algae are divided into microalgae and macroalgae or seaweed. Focusing on macroalgae, they are further classified into three main groups: green (Chlorophyta), brown (Phaeophyta) and red algae (Rhodophyta). Thousands of species are found in these three subgroups, many of them being of food interest [1]. In addition, they have been used in traditional medicine for many years against various diseases (tuberculosis, 
arthritis, colds and influenza) [2,3]. Seaweeds are part of the diet in many cultures, especially in Asia since they present a high nutritional value. Algae are a valuable source of protein (5-14\%), fiber, lipids $(<3 \%)$ and carbohydrates (13-19\%), in addition to its content in micronutrients (such as salts, minerals and vitamins) necessary for the correct and healthy functioning of the organism $[4,5]$. Likewise, they are also rich in several secondary metabolites such as phenolic compounds, phycobiliproteins, carotenoids, alkaloids, terpenes, sulfated polysaccharides or phytosterols [6,7]. For these reasons, algae have been attracting curiosity also in the pharmaceutical industry for a few years, as they are very varied natural matrices that contain a great diversity of compounds with bioactive capabilities. Some of those beneficial biological functions to human health are antioxidant, anti-inflammatory, cardioprotective, antimicrobial, antifungal, antiviral and anticancer activities, among others [8]. Phytosterols extracted from various algae have demonstrated antimicrobial, analgesic and antioxidant functions, fucosterol being the predominant sterol in brown algae [4,9]. Other well-known molecules present in algae are sulfated polysaccharides which, in addition to presenting capabilities as gelling and thickening agents such as alginates and carrageenan, are associated with antioxidant, anticoagulant, antiviral, antitumor, anti-inflammatory and immunostimulant properties, such as the fucoidans present in the cell walls and the extracellular matrices of algae, especially the brown ones [10]. These reasons make them of great interest to the healthcare and food industries [11,12]. Phenolic compounds are associated with a strong antioxidant capacity, which is also associated with other beneficial properties such as anti-inflammatory, antimicrobial and anticancer. Phenolic compounds constitute a very heterogeneous group of molecules that can be classified in different ways. Notable among them are flavonoids, phenolic acids, lignans and tannins [13]. Within this last group are classified the so-called phlorotannins, molecules whose structure corresponds to polymers of phloroglucinol (1,3,5-trihydroxybenzene), which are present exclusively in algae, especially in brown. Phlorotannins can be deeper divided into four major groups (fuhalols and phlorethols, fucols, fucophloroethols and eckols), and are associated with powerful biological activities such as antioxidant, anti-inflammatory, antimicrobial, antifungal, antibiofilm or antifouling, hepatoprotective and antiviral $[14,15]$. Moreover, some fatty acids such as palmitic acid, linoleic acid, linolic acid, palmitoleic acid, eicosapentaenoic acid, stearic acid, or oleic acid have also demonstrated cardioprotective, antitumor and antimicrobial activities [16,17].

As described, the compounds contained in algae show numerous activities beneficial to health. Regarding the antimicrobial capacity of algae extracts, they have been successfully analyzed against various pathogens of great concern to human health. Some of the Gram positive bacteria studied are strains of Bacillus subtilis, Bacillus cereus, Staphylococcus aureus, Enterococcus faecalis or Micrococcus luteus, while the Gram negative bacteria analyzed include Klebsiella pneumoniae, Serratia marcescens, Escherichia coli, Pseudomonas aeruginosa, Salmonella Typhimirium or Vibrio cholerae [18]. The antimicrobial mechanisms of action associated with compounds extracted from marine algae are varied. These include the changes that occur in the permeability of the membranes thanks to the interaction with proteins and lipids present in them, or the ability to inhibit enzymes $[17,19]$. Some examples of specific compounds extracted from algae that have shown antimicrobial function are phlorotannins, laminarin, sargafuran, peyssonoic acid, bromophycolides, neurymenolides, acetylmajapolene and phycobiliproteins, phytol, fucosterol, neophytadiene, palmitic, palmitoleic and oleic acids [20,21].

The main current applications of algae include their use as fertilizers, in the production of biofuels, in the cosmetic industry and, of course, in food and agriculture [5,22]. An example of the application of compounds with antimicrobial capabilities obtained from macroalgae is their use in the livestock industry as food supplements, since it is a good strategy to reduce the use of antibiotics in animal feed. This substitution of antibiotics by natural functional molecules would help to combat the current growing resistance to antibiotics by pathogenic microorganisms $[14,20]$. Therefore, this abundant and ubiquitous source of bioactive compounds represented by algae could be used to obtain extracts rich in such antimicrobial compounds to be applied in the agri-food, cosmetic or pharmaceutical industries. 


\section{Microbial Contamination in Food Industry}

Feeding habits are undergoing drastic changes since consumers are increasingly searching for natural food ingredients. Synthetic additives are generating rejection due to the harmful health effects associated with them, such as certain neurological or immune conditions (hyperactivity, allergies, etc.). This situation has triggered the research for new natural sources of bioactive compounds that can exert actions that improve or extend the shelf life of products, such as preservative function, among others, which has become a trending topic for the food industry [21].

On the other hand, the appearance of microbial contamination in food products during their processing, storage, distribution and consumption is a sanitary problem that the mentioned industry also must face. This contamination may be favored by intrinsic factors in food, such as bacterial load or its water content, or by external factors that condition the stability of the food, such as the presence of oxygen, humidity or temperature of the environment [23]. To avoid this contamination that can lead to foodborne illnesses, different food safety control strategies are carried out such as hazard analysis and critical control point (HACCP), sanitation standard operating procedure (SSOP) and good manufacturing practices (GMP), the first being the most widely used globally. However, these strategies also have drawbacks such as the detection and determination of the critical control points. In this regard, novel techniques that allow contamination control while guaranteeing food safety are required. These controls are necessary to prevent specific contaminations that can lead to cross-contamination throughout the food production and consumption chain [24].

Some typical food contaminants are Pseudomonas sp., Listeria monocytogenes, Salmonella sp., Shigella flexneri, V. cholerae, E. coli, Bacillus sp. and Campylobacter jejuni. Additionally, many of the pathogens responsible for contaminations that lead to foodborne illnesses can form biofilms, making them even more harmful and difficult to control. There are various techniques to sterilize food so that microbial contamination can be avoided, such as the use of ozone, electrical pulses, ultrasounds, microwaves, radiation or thermal processes [25]. However, as aforementioned, algae are rich in bioactive compounds such as alkaloids, terpenoids, polysaccharides, pigments, cyclic peptides, phenols, lipid, and vitamins [20], many of which show antimicrobial and anti-biofilm properties that have been successfully tested against some of the common food pathogens. For this reason, compounds obtained from marine algae that show antimicrobial capabilities could be used alternatively, since their use would be doubly advantageous, both to mitigate microbial contamination in food and to replace certain synthetic additives, increasing the attractiveness of the products to consumers, their value and being healthier since synthetic preservatives could be replaced [20,26].

This work collects information about natural compounds extracted from marine algae that show antimicrobial capabilities against pathogenic bacteria commonly responsible for food contamination that can cause foodborne illnesses. Furthermore, potential applications of these bioactive compounds in the food industry are described, discussing their compliance with quality and safety controls, and finishing with some future possible approaches.

\section{Antibacterial Potential of Bioactive Compounds from Macroalgae against Pathogens and Spoilage Microorganisms}

\subsection{Evaluation Criteria}

Agar diffusion methods are frequently used to evaluate the antimicrobial capacity of new compounds. In this microbiological technique, agar plates are inoculated with a standardized inoculum of the tested microorganism. Then, filter paper discs containing the tested compound at the desired concentration are placed on the agar surface. Generally, the antimicrobial agent diffuses into the agar and inhibits germination and growth of the tested microorganism and then the diameters of inhibition growth zones are measured [27]. Nevertheless, there is no standard way to carry out a qualitative evaluation. Furthermore, different authors have used different scales [28-31]. Therefore, to standardize the classification, this review will adopt the classification proposed by the Clinical and 
Laboratory Standards Institute (CLSI). Depending on the measured diameter of the inhibition growth zone, the bacteria can be categorized as susceptible ( $\geq 20 \mathrm{~mm})$, intermediate (I, 15-19 $\mathrm{mm}$ ) or resistant $(\mathrm{R}, \leq 14 \mathrm{~mm})$ [32].

Another standard way to evaluate the antimicrobial susceptibility of extracts or purified compounds is the broth dilution test. In this experimental approach, the test substance is diluted in a broth or saline solution and added to the broth containing antimicrobial agent, the microorganism in the mixture can grow for 16 to $24 \mathrm{~h}$. The result is normally quantitative and expressed as minimal inhibition concentration $[33,34]$.

\subsection{Gram Negative}

\subsubsection{Enterobacteriaceae}

Members of the Enterobacteriaceae family possess Gram-negative stain properties and they are facultative anaerobes, fermenting sugars to produce lactic acid and other various end products. The family Enterobacteriaceae includes many bacteria that are found in the human or animal intestinal tract, including human pathogens. Enterobacteriaceae are useful indicators of hygiene and post-processing contamination of heat-processed foods.

\section{Escherichia coli}

Escherichia coli is an oxidase-negative rod-shaped bacterium. This bacterium grows preferably at a temperature of $37^{\circ} \mathrm{C}$ and can either be nonmotile or motile, presenting in its constitution peritrichous flagella. Even though E. coli can be a harmless resident in the gastrointestinal tract, it also has the pathogenic capacity to cause significant diarrheal and extraintestinal diseases. Pathogenic variants of E. coli cause significant morbidity and mortality worldwide [35]. One of the most important sources of foodborne diseases worldwide is caused by the Shiga-type toxins produced by the E. coli strains serotype O157:H7. These strains, in particular, started to be considered as a significant risk to public health in the 1980s, when they were associated with outbreaks of gastrointestinal symptoms and hemolytic uremic syndrome [36]. In 2018, in the European Union, a total of 2.28 citizens per 100,000 habitants were contaminated with E. coli related food poisoning [37]. In the years between 2003 and 2012, the United States recorded 390 E. coli O157 type outbreaks, with 65\% of them being food-related [38].

Most of the E. coli associated foodborne outbreak cases over the past decade have been attributed to the consumption of uncooked foods (raw source or during the preparation process) contaminated by pathogenic E. coli. Furthermore, E. coli did not only cause huge economic losses but also impacted human health and even caused death [38,39]. E. coli is widely selected as a target microorganism to assess the antimicrobial activity of compounds and crude extracts from numerous macroalgae species (Table 1). In this review, it was found that the most tested E. coli strain was the American Type Culture Collection (ATCC) 25922 [40-53]. Nevertheless, methanolic extracts of the brown algae Scytosiphon lomentaria and Padina pavonica inhibited the growth (diameter $>15 \mathrm{~mm}$ ) of the E. coli erotype O157:H7 [54].

The most studied species are brown algae, followed by the red and finally the green algae. As it can be seen in Table 1, brown algae [28,50,54-60] promoted inhibition growth zones larger than $15 \mathrm{~mm}$ diameter, allowing the antibacterial activity categorization of intermediate or susceptible. Minimal inhibition concentration (MIC) values ranging from $7.5 \mathrm{mg} / \mathrm{L}$ to $0.16 \mathrm{mg} / \mathrm{L}$ were achieved when an acetone-based crude extract of Cutleria multifida was used [42]. In the red algae group, the Gracilaria multipartite [56,58], Corallina officinalis [50], G. corticata [61] and Rhodomela confervoides [60] were the algae that presented antibacterial activity between intermediate to susceptible against E. coli. Moreover, the alga Laurencia papillosa contains several bioactive molecules such as pigments (chorophyll, carotenoids, phycocyanine, phycoerythrine), proteins, carbohydrates, phenolics, flavonoids, tannin, fatty acids in its composition, presenting the lowest MIC $(0.2 \mathrm{mg} / \mathrm{mL})$ [62]. 
Table 1. Antimicrobial effect of algae extracts in Escherichia coli (reference strains and serotypes: American Type Culture Collection (ATCC) 8739/10536/11775/25922/35218; MTCC 739; NCIMB 50034; DSM 498; RCM 01002 52-6; PTCC1330, O157:H7; O126:B16).

\begin{tabular}{|c|c|c|c|c|}
\hline Seaweed & Solvent & $\mathrm{MIC} / \mathrm{IC} 50 / \mathrm{MBC}(\mathrm{mg} / \mathrm{mL})$ & Agar Diffusion & Ref. \\
\hline Laurencia brandenii (R) & $\mathrm{MeOH}$ & & $\operatorname{Re}$ & [63] \\
\hline G. ornata (R) & $\mathrm{H}_{2} \mathrm{O} / \mathrm{EtOH} \mathrm{f}$ & & $\mathrm{NI} / \mathrm{NI} / \mathrm{R}$ & [64] \\
\hline Pterocladia capillacea (R) & \multirow{2}{*}{$\mathrm{H}_{2} \mathrm{O}_{\text {cold } /} \mathrm{H}_{2} \mathrm{O}_{\text {hot }}$} & & $\mathrm{NI} / \mathrm{NI}$ & \multirow[t]{2}{*}[55]{} \\
\hline Dictyopteris membranacea (B) & & & $\mathrm{I} / \mathrm{NI}$ & \\
\hline D. membranacea (B) & $\begin{array}{c}(\mathrm{MeOH}: \mathrm{DCM}) / \mathrm{MeOH} \mathrm{f} / \mathrm{AcO} \\
\text { f/(MeOH:DCM) }\end{array}$ & & $\operatorname{Re} / \operatorname{Re} / \mathrm{I} / \mathrm{NI}$ & [28] \\
\hline P. capillacea (R)/Osmundaria obtusiloba (R) & $\mathrm{EtOH} / \mathrm{Hex}$ & & $\mathrm{NI}$ & [40] \\
\hline $\begin{array}{c}\text { Dictyota dichotoma }(\mathrm{B}) / \text { P. pavonica }(\mathrm{B}) / \text { Sargassum } \\
\text { vulgare }(\mathrm{B})\end{array}$ & $\mathrm{AcO}$ & $\operatorname{MIC}(2.5 / 2.5 / 5)$ & & [41] \\
\hline $\begin{array}{c}\text { Cystoseira amentacea (B)/Cystoseira barbata } \\
\text { (B)/Cystoseira compressa (B) }\end{array}$ & $\mathrm{AcO}$ & MIC (5) & & [46] \\
\hline $\begin{array}{c}\text { Cystoesira myrica (B)/Cystoesira trinodis } \\
\text { (B)/Padina gymnospora (B)/Sargassum } \\
\text { dentifolium (B)/Sargassum hystrix } \\
\text { (B)/Actinotrichia fragilis (R)/Caulerpa racemosa } \\
\text { (G)/Codium fragile (G) }\end{array}$ & $\mathrm{MeOH} / \mathrm{EtAc}$ & & $\operatorname{Re} / \operatorname{Re}$ & [47] \\
\hline Padina australis Hauck (B) & \multirow{6}{*}{$\mathrm{MeOH} / \mathrm{DCM} / \mathrm{n}-\mathrm{Hex}$} & MIC $(0.88 / 1.04 / 1.25)$ & & \multirow{6}{*}[48]{} \\
\hline Sargassum polycystum (B) & & MIC $(0.88 / 1.04 / 1.04)$ & & \\
\hline S. polycystum (B) & & $\operatorname{MIC}(0.73 / 0.83 / 0.73)$ & & \\
\hline P. australis Hauck (B) & & MIC (2.08/2.08/1.66) & & \\
\hline S. polycystum (B) & & MIC (0.83/1.04/0.625) & & \\
\hline S. polycystum (B) & & $\operatorname{MIC}(0.52 / 0.83 / 0.42)$ & & \\
\hline Gracilaria sp. (R) & $\mathrm{MeOH}$ & & $\operatorname{Re}$ & [65] \\
\hline L. papillosa (R) & DCM/(DCM:MeOH)/MeOH/ $\mathrm{H}_{2} \mathrm{O}$ & $\begin{array}{l}\text { MIC }(0.8 / 0.2 / 0.8 / 0.4) \\
\operatorname{MBC}(1.4 / 0.2 / 1.6 / 0.6)\end{array}$ & $\mathrm{Re} / \mathrm{Re} / \operatorname{Re} / \operatorname{Re}$ & {$[62]$} \\
\hline
\end{tabular}


Table 1. Cont.

\begin{tabular}{|c|c|c|c|c|}
\hline Seaweed & Solvent & MIC/IC50/MBC (mg/mL) & Agar Diffusion & Ref. \\
\hline Padina antillarum (B) & \multirow{3}{*}{$\mathrm{MeOH} / \mathrm{EtAc}$} & $\operatorname{MIC}(3.75 / 3.75)$ & $\mathrm{I} / \mathrm{I}$ & \multirow{3}{*}{ [66] } \\
\hline Padina boergeseni (B) & & MIC (7.5/7.5) & $\mathrm{I} / \mathrm{I}$ & \\
\hline Ulva flexuosa $(\mathrm{G})$ & & MIC (1.18/0.93) & $\mathrm{S} / \mathrm{S}$ & \\
\hline $\begin{array}{l}\text { Ulva lactuca }(\mathrm{G}) / \text { Enteromorpha intestinales } \\
(\mathrm{G}) / \text { Cladophora vagabunda }(\mathrm{G})\end{array}$ & $\mathrm{EtOH}$ & & $\mathrm{R} \mathrm{e} / \operatorname{Re} / \mathrm{I}$ & [67] \\
\hline Grateloupia doryphora (R) & $\mathrm{EtOH} / \mathrm{MeOH} / \mathrm{EtAc}$ & & NI & [68] \\
\hline $\begin{array}{l}\text { Halimeda tuna }(\mathrm{G}) / \text { Melanothamnus afaghusainii } \\
\text { (R)/Dictyota indica (B)/D. dichotoma var. intricata } \\
\text { (B)/Sargassum lanceolatum (B) }\end{array}$ & $\mathrm{EtOH}$ & & $\operatorname{Re}$ & [57] \\
\hline C. trinodis (B) & DiEt:EtOH:Hex & MIC (4.12) & & [49] \\
\hline Cladophora glomerata (G) & \multirow{7}{*}{$\mathrm{EtOH}$} & $\operatorname{MIC}(>1.25)$ & I & \multirow{7}{*}[50]{} \\
\hline Enteromorpha linza $(\mathrm{G})$ & & $\operatorname{MIC}(>5)$ & $\operatorname{Re}$ & \\
\hline Ulva rigida $(\mathrm{G})$ & & $\operatorname{MIC}(>10)$ & $\operatorname{Re}$ & \\
\hline C. barbata (B) & & $\operatorname{MIC}(>5)$ & $\operatorname{Re}$ & \\
\hline P. pavonica (B) & & $\operatorname{MIC}(>1.25)$ & I & \\
\hline C. officinalis (R) & & $\mathrm{MIC}(>1.25)$ & I & \\
\hline Ceramium ciliatum (R) & & $\operatorname{MIC}(>5)$ & $\operatorname{Re}$ & \\
\hline D. membranacea (B) & $\mathrm{H}_{2} \mathrm{O} / \mathrm{EtAc} / \mathrm{CHCl}_{3}$ & & NI & [59] \\
\hline $\begin{array}{l}\text { Cymodocea nodosa }(\mathrm{G}) / \text { H. tuna }(\mathrm{G}) / \text { C. barbata } \\
\text { (B)/Codium bursa }(\mathrm{G})\end{array}$ & DCM:MeOH & & NI & [51] \\
\hline $\begin{array}{c}\text { Chaetomorpha linum (G)/Cladophora rupestris } \\
\text { (G)/Fucus serratus (B)/Halosiphon tomentosus } \\
\text { (B)/Saccharina latissima (B)/Bonnemaisonia } \\
\text { hamifera (R)/Callithamonion corymbosum } \\
\text { (R)/Ceramium tenuicorne (R)/Dasya baillouviana } \\
\text { (R)/Delesseria sanguinea (R)/Dumontia contorta } \\
\text { (R)/Polysiphonia elongata (R)/Polysiphonia nigra } \\
\text { (R)/R. confervoides (R) }\end{array}$ & DCM & & NI & [69] \\
\hline
\end{tabular}


Table 1. Cont

\begin{tabular}{|c|c|c|c|c|}
\hline Seaweed & Solvent & MIC/IC50/MBC (mg/mL) & Agar Diffusion & Ref. \\
\hline G. corticata (R) & $\mathrm{MeOH} / \mathrm{DMSO}$ & & $\mathrm{S} / \operatorname{Re}$ & \multirow{2}{*}[61]{} \\
\hline G. edulis (R) & $\mathrm{MeOH}$ & & $\operatorname{Re}$ & \\
\hline $\begin{array}{l}\text { S. lomentaria (B)/P. pavonica (B)/Cystoseira } \\
\text { mediterranea (B)/Hypnea musciformis } \\
(\mathrm{R}) / \text { Spyridia filamentosa }(\mathrm{R})\end{array}$ & $\mathrm{MeOH}$ & & $\mathrm{I} / \mathrm{I} / \mathrm{NI} / \mathrm{NI} / \operatorname{Re}$ & [54] \\
\hline $\begin{array}{l}\text { A. fragilis (R)/C. myrica (B)/Hormophysa } \\
\text { cuneiformis (B)/L. papillosa (R)/Sargassum } \\
\text { cinereum (R)/Turbinaria turbinata (B) }\end{array}$ & $\mathrm{MeOH}$ & & $\operatorname{Re}$ & {$[52]$} \\
\hline C. barbata (B) & $\mathrm{EtOH}$ & & $\operatorname{Re}$ & [70] \\
\hline Kappaphycus alvarezii (R) & $\mathrm{EtOH} / \mathrm{Hot} \mathrm{H}_{2} \mathrm{O}$ & & $\mathrm{NI}$ & [71] \\
\hline Oedogonium sp. (G) & \multirow{4}{*}{$\mathrm{MeOH} / \mathrm{EtOH}$} & & NI & \multirow{4}{*}[72]{} \\
\hline Stigeoclonium sp. (G) & & & $\mathrm{I} / \mathrm{I}$ & \\
\hline Ulothrix sp. (G) & & & $\mathrm{I} / \mathrm{I}$ & \\
\hline Nitzschia sp. (G) & & & $\operatorname{Re} / \mathrm{I}$ & \\
\hline \multirow{2}{*}{ Bifurcaria bifurcata (B) } & \multirow{2}{*}{$\mathrm{MeOH} / \mathrm{DCM}$} & & $\mathrm{Re} / \mathrm{NI}$ & \multirow{2}{*}[53]{} \\
\hline & & & $\operatorname{Re} / \operatorname{Re}$ & \\
\hline Hypnea flagelliformis (R) & \multirow{3}{*}{$\mathrm{MeOH} / \mathrm{H}_{2} \mathrm{O} / \mathrm{DCM}$} & & NI & \multirow{3}{*}[73]{} \\
\hline C. myrica (B) & & & $\mathrm{NI}$ & \\
\hline Sargassum boveanum $(\mathrm{B})$ & & & $\mathrm{NI}$ & \\
\hline Turbinaria conoides (B) & $\mathrm{n}-\mathrm{Hex} / \mathrm{ClHx} / \mathrm{MeOH} /\left(\mathrm{EtOH}: \mathrm{H}_{2} \mathrm{O}\right)$ & & $\mathrm{Re} / \mathrm{Re} / \mathrm{NI}$ & [74] \\
\hline $\begin{array}{c}\text { Cystoseira tamariscifolia }(\mathrm{B}) / \text { P. pavonica }(\mathrm{B}) / \mathrm{R} \text {. } \\
\text { confervoides }(\mathrm{R}) / \text { U. lactuca }(\mathrm{G})\end{array}$ & $\mathrm{MeOH}$ & & $\mathrm{I} / \mathrm{I} / \mathrm{I} / \mathrm{Re}$ & {$[60]$} \\
\hline $\begin{array}{l}\text { Laurencia obtusa (R)/Codium elongatum (G)/C. } \\
\text { multifida (B) }\end{array}$ & $\mathrm{AcO}$ & MIC $(0.17 / 2.5 / 0.16)$ & & [42] \\
\hline Fucus vesiculosus (B)/Cystoseira baccata (B) & $\mathrm{EtOH}$ & IC50 $(2.25 / 2.5)$ & & [43] \\
\hline Saccharina japonica (B) & $\mathrm{CO}_{2}$ & MIC (3.4) & & [44] \\
\hline
\end{tabular}


Table 1. Cont

\begin{tabular}{|c|c|c|c|c|}
\hline Seaweed & Solvent & MIC/IC50/MBC (mg/mL) & Agar Diffusion & Ref. \\
\hline Enteromorpha prolifera (G) & \multirow{7}{*}{$\mathrm{PeEt} / \mathrm{DiEt} / \mathrm{EtAc} / \mathrm{MeOH}$} & & $\mathrm{I} / \mathrm{I} / \mathrm{R}$ e/I & \multirow{7}{*}{ [58] } \\
\hline Ulva reticulata $(\mathrm{G})$ & & & $\mathrm{S} / \mathrm{I} / \mathrm{Re} / \mathrm{I}$ & \\
\hline C. myrica (B) & & & I & \\
\hline P. pavonica (B) & & & I & \\
\hline Turbinaria triquetra (B) & & & I & \\
\hline Sargassum portieriatum (B) & & & $\mathrm{S} / \mathrm{I} / \mathrm{I} / \mathrm{I}$ & \\
\hline G. multipartite (R) & & & $\mathrm{I} / \mathrm{I} / \mathrm{I} / \operatorname{Re}$ & \\
\hline Hypnea esperi (R)/Caulerpa prolifera (G) & $\mathrm{MeOH}$ & & $\mathrm{Re} / \mathrm{NI}$ & [75] \\
\hline Himanthalia elongata (B) & $\mathrm{n}-\mathrm{Hex} / \mathrm{EtOH} / \mathrm{H}_{2} \mathrm{O}$ & $\operatorname{MBC}(7.2 / 6.0 / 12.5)$ & & [21] \\
\hline $\begin{array}{l}\text { Sargassum ilicifolium (B)/H. cuneiformis (B)/S. } \\
\text { polycystum (B)/Sargassum sp. (B)/T. conoides } \\
\text { (B)/Turbinaria decurrens (B)/Turbinaria ornata (B) }\end{array}$ & $\mathrm{MeOH}$ & & $\operatorname{Re}$ & [76] \\
\hline Sargassum incisifolium(B) & EtAc & & $\operatorname{Re}$ & [76] \\
\hline L. complanata $(\mathrm{R})$ & $\mathrm{MeOH} / \mathrm{Hex} \mathrm{f/(Hex:EtAc)} \mathrm{f/MeOH} \mathrm{f}$ & & $\mathrm{Re} / \mathrm{NI} / \mathrm{NI} / \mathrm{NI}$ & \multirow{3}{*}{ [77] } \\
\hline $\begin{array}{c}\text { Grateloupia sp. (R)/G. corticata (R)/Spyridia sp. } \\
\text { (R)/Meta-mastophora sp. (R)/Calloseris sp. } \\
(\mathrm{R}) / \text { Neurymenia fraxinifolia }(\mathrm{R})\end{array}$ & $\mathrm{MeOH}$ & & $\operatorname{Re}$ & \\
\hline Halymenia sp. (R) & DiEt & & $\operatorname{Re}$ & \\
\hline U. reticulata $(\mathrm{G})$ & $\left(\mathrm{MeOH}: \mathrm{CHCl}_{3}: \mathrm{H}_{2} \mathrm{O}\right)$ & & I & [78] \\
\hline \multirow{2}{*}{ U. rigida $(\mathrm{G})$} & \multirow{2}{*}{$\begin{array}{c}\left(\mathrm{EtOH}: \mathrm{H}_{2} \mathrm{O}\right) / \mathrm{Hex} \mathrm{f} / \mathrm{CHCl}_{3} \mathrm{f} / \mathrm{EtAc} \\
\text { f/ButOH f/ } \mathrm{H}_{2} \mathrm{O} \mathrm{f}\end{array}$} & & $\mathrm{Re} / \mathrm{NI} / \mathrm{I} / \mathrm{Re} / \mathrm{NI} / \mathrm{NI}$ & \multirow{2}{*}{ [45] } \\
\hline & & & $\mathrm{NI} / \mathrm{Re} / \mathrm{NI} / \mathrm{NI} / \mathrm{NI}$ & \\
\hline \multirow[t]{2}{*}{ U. lactuca $(\mathrm{G}) / D$. dichotoma $(\mathrm{B})$} & \multirow{2}{*}{$\mathrm{MeOH} / \mathrm{DiEt} / \mathrm{CHCl}_{3}$} & & $\operatorname{Re} / \operatorname{Re} / S$ & \multirow{2}{*}{ [79] } \\
\hline & & & $\operatorname{Re} / \operatorname{Re} / \operatorname{Re}$ & \\
\hline U. rigida $(G)$ & DCM/DCM:MeOH & & $\mathrm{NI} / \operatorname{Re}$ & [80] \\
\hline
\end{tabular}


In the Chlorophyta algal group, the U. flexuosa [66], C. vagabunda [67], U. lactuca [56,79], G. multipartite, C. glomerata [50]; Hypnea valentiae [56], Stigeoclonium sp., Ulothrix sp., Nitzschia sp. [72], E. prolifera, $U$. reticulata [81] and $U$. rigida [45] showed the most promising antibacterial capacity since they were able to inhibit $E$. coli growth in the intermediate or susceptible category. U. flexuosa [66] produced diameters of the inhibition growth zone larger than $20 \mathrm{~mm}$ and MICs of 1.18 and $0.93 \mathrm{mg} / \mathrm{mL}$ for the methanolic and ethyl acetate extracts, respectively. Studies made on S. polycystum and P. australis by Hauck [48] showed that crude extracts of algae collected from different locations gave slightly different MICs. In addition, some research made using the red algae G. multipartite showcased that there was a connection between the season when the specimen was collected and its antimicrobial effect against E. coli [56]. This effect was further proven by an antibiogram made using the algae $U$. rigida [80]. The main technique used to obtain bioactive compounds from algae is maceration using different solvents, even though chemicals such as chloroform are still used, the majority of studies use methanol, ethanol and water, as shown in Table 1. The solvent effect of Nitzschia sp. and Stigeoclonium sp. ethanolic extracts on E. coli growth leads to a higher diameter of the inhibition growth zone than methanolic ones, but no significant difference was found when Ulothrix sp. extracts were used. The findings reported on the antibacterial activity from marine algae extracts from the Red Sea extracted with different solvents implied that petroleum ether, diethyl and ethyl acetate were the best extractive solvents for extracting bioactive compounds (catechin, phlorotannins flavonols, glycosides flavonol and phenolic compounds), followed by methanol [72]. On the other hand, chloroform-based crude extracts of $U$. lactuca presented a higher antibacterial activity than diethyl ether and methanol ones. However, in the same study, D. dichotoma methanolic extract displayed better antimicrobial activity [79]. This implies that solvent selection is critical and should be optimized for each alga.

\section{Enterobacter spp.}

Enterobacter spp. are foodborne pathogens responsible for opportunistic infections. The genus Enterobacter consist of 12 species, including E. cloacae and E. aerogenes-the most commonly isolated species that cause human infections [82]. Studies were conducted to examine the effects of the temperature and relative humidity on the survival of E. aerogenes on stainless steel, polyvinyl chloride and ceramic tile. Although surface type had little effect on bacteria survival, the temperature had a clear effect on the E. aerogenes survival. In this case, bacterial growth presented its maximum at $7^{\circ} \mathrm{C}$ and $15^{\circ} \mathrm{C}$ and $50 \%$ relative humidity on all surfaces [83]. Moreover, in 46 pepper samples from Vietnam, the E. cloacae were isolated in 30 samples (65.2\%) [84].

Concerning the antagonistic activity of algae extracts against Enterobacter spp., the literature is scarce as shown in Table 2. The most common alga group tested is Phaeophytha phylum. However, all extracts tested were classified as resistant or present with no antimicrobial activity against Enterobacter spp. On the other hand, the outcome obtained with purified ulvan, a cell wall polysaccharide formed by bioactive molecules such as rhamnose, galactose, xylose, mannose and glucose, uronic acid and sulphate groups, from the green alga $U$. reticulata excels with a report of a diameter of the inhibition growth zone of $20 \mathrm{~mm}$ [78]. It is also interesting to bring to light that in the range of algae shown in Table 2, $U$. reticulata is not only the one that shows the best results but also is the only green algae tested.

\section{Shigella flexneri}

Shigella spp. is an etiological agent of bacillary dysentery or shigellosis. This disease is marked by fever, violent abdominal cramps and rectal urgencies [85]. Shigella spp. causes annually approximately 500,000 illnesses, 6000 hospitalizations and 40 deaths in the United States [85]. S. dysenteria is usually present in the epidemic and outbreak forms of dysentery disease. S. flexneri and S. sonnei are mainly responsible for endemic shigellosis disease in developing and developed nations, respectively. While S. boydii is restricted to India and neighboring countries, the S. flexneri was found to be the most commonly isolated species (68\%) in a multi-centric study from six Asian countries including China, Vietnam, Thailand, Bangladesh, Pakistan and Indonesia [86]. 
Unfortunately, Shigella spp. have presented resistance to most used antibiotics, which is a serious concern to health [87]. Usually, food is contaminated through Shigella spp. by an infected food handler who practices poor personal hygiene. Moreover, Shigella spp. is acid resistant, salt-tolerant and can survive at infective levels in many types of foods such as fruits and vegetables, low $\mathrm{pH}$ foods, prepared foods and foods held in a modified atmosphere or vacuum packaging [85]. Studies involving brown algal extracts against $S$. flexneri were conducted $[76,88]$ and the majority of the tested algae species belonged to the Sargassum taxa, as shown in Table 2. From observing Table 2, we can notice that all the antimicrobial test results were attained by Kirby-Bauer test and presented no quantitative results. Nevertheless, the capacity of different solvents to extract bioactive compounds (indoles, terpenes, acetogenins, phenols, fatty acids and volatile halogenated hydrocarbons, and also high percentage of octadecadienoic acid, eicosane, dotriacontane, tritetracontane, docosane, octatriacontyl, heptacosane and tetracosane) [88] able to promote the inhibition growth of S. flexneri was evaluated [88] and the highest inhibition was obtained with $S$. vulgare methanol $(\mathrm{MeOH})$ and diethyl (DiEt) crude extracts; Sargassum fusiforme $\mathrm{DiEt}>$ chloroform $\left(\mathrm{CHCL}_{3}\right)>$ ethanol $(\mathrm{EtOH}) ;$. pavonica $\mathrm{EtOH}>\mathrm{CHCL}_{3}>\mathrm{DiEt}$ and $\mathrm{MeOH}$; Ceramium rubrum $\mathrm{EtOH}>\mathrm{CHCL}_{3}$ DiEt and $\mathrm{MeOH}$.

\section{Proteus spp.}

Proteus spp. microorganisms are widely distributed in the natural environment, including water, soil and manure. Due to their proteolytic activity, the ability to hydrolyze urea to ammonia and carbon dioxide, as well as the oxidative deamination of amino acids, these bacteria are involved in the decomposition of organic matter of animal origin [89]. Currently, the genus Proteus is divided into Proteus mirabilis, P. vulgaris, P. penneri, P. hauseri. These bacteria are known to be human opportunistic pathogens, isolated from urine, wounds and other clinical sources [90]. Some P. mirabilis strains were found to be associated with food poisoning outbreaks [91]. This bacterium has been found in several food animals such as poultry, especially chickens [92,93]. Ten prepared vegetable salads obtained at various sales points in Ekpoma, Nigeria were investigated for the isolation of these bacteria. The results demonstrate the presence of Proteus sp. in $20 \%$ of the samples [94]. New multiple resistant forms of this bacteria have been discovered. Therefore, it may become a major food issue in the future [95]. P. vulgaris exhibited the highest resistance to the antibiotics, with a value of six out of eleven antibiotics $(54.5 \%)$ [96].

In the last decade, most of the studies on algal antimicrobial compounds include (Table 2) Proteus spp., namely the strains of $P$. vulgaris $[97,98]$ or P. mirabilis $[29,62,99,100]$. The brown algae $T$. conoides, P. gymnospora and Sargassum tenerrimum were extracted with various solvents: methanol, acetone, petroleum ether, ethanol, ethyl acetate, chloroform and diethyl ether. The obtained crude extracts were tested against Proteus sp. The most favorable inhibition results were achieved with $T$. conoides acetone extract, methanolic- and chloroform-based extracts of P. gymnospora and petroleum ether extract of S. tenerrimum [101]. Purified extracts from several brown algae, rich in phlorotannins, evidenced antimicrobial activity against several pathogens including P. mirabilis [102]. A MIC of $0.65 \mathrm{mg} / \mathrm{mL}$ was obtained with the n-butanol fraction of Liagora hawaiiana extract [97]. Epitaondiol, epitaondiol diacetate, epitaondiol monoacetate, stypotriol triacetate, 14-ketostypodiol diacetate and stypodiol were successfully isolated from the dichloromethane (DCM) crude extract of brown alga Stypopodium flabelliforme and these compounds demonstrated antimicrobial activity against $P$. mirabilis (MICs below $1 \mathrm{mg} / \mathrm{mL})$ [103].

\section{Salmonella spp.}

Salmonellae are flagellated organisms that are ubiquitously distributed and cause salmonellosis. Non-typhoidal salmonellosis is a worldwide disease of humans and animals. Animals are the main reservoir, and the disease is usually foodborne, although it can be spread from person to person [104]. Around the world, several outbreaks of food poisoning related to Salmonella are documented, e.g., an outbreak of infection with non-typhoidal Salmonella affecting 396 patients in the community was 
associated with frozen pies supplied for cooking by the consumer in a microwave oven [105]. During a prevalence study made in Asia involving 807 meat samples, 159 (19.7\%) were positive for Salmonella. Pork had the highest prevalence $(37.3 \%, 107 / 287)$ of Salmonella contamination, followed by beef (16.1\%, 26/161), mutton $(10.9 \%, 10 / 92)$, dumplings $(6.6 \%, 14 / 212)$ and smoked pork $(3.6 \%, 2 / 55)$ [106]. A report focusing on multidrug-resistant Salmonella in the United States in years 2018-2019 linked 255 infections to contaminated beef and soft cheese [107]. From a work focused on chickens, a total of 384 caecal content samples were collected for microbiological examination and the overall prevalence of Salmonella was $14.6 \%$ [108], showing the ubiquity of this bacteria.

Overall, the most studied salmonella species in antimicrobial tests performed with alga extracts is Salmonella Typhi. Studies made with these bacteria species allow the classification of the antimicrobial activity of intermediate to susceptible (diameter of the inhibition growth zone higher than $15 \mathrm{~mm}$ ) from the Phaeophyta phylum: D. membranacea [28], P. gymnospora [47], D. dichotoma var intricate (B), D. indica (B) [57], S. lomentaria (B) [54], and Ecklonia cava [109]. Among the Chlorophytes and Rhodophytes, U. rigida and C. officinalis [50] also present antagonistic effects to S. Typhi. Alga extracts were also tested against additional Salmonella spp.: S. Enteridis, S. Choleraesuis and S. Gallinarum. Although several extracts presented no activity in Table 2, the ethyl acetate fraction extract of E. cava presented a MIC of $0.25 \mathrm{mg} / \mathrm{mL}[109]$.

\subsubsection{Pseudomonadaceae: Pseudomonas aeruginosa}

Pseudomonas aeruginosa is an encapsulated and rod-shaped bacterium that is citrate, catalase and oxidase positive. P. aeruginosa can be found in water, soil, skin flora and most man-made environments throughout the world. This bacterial pathogen can cause broad human illnesses comprising osteomyelitis, endocarditis, pneumonia, meningitis, septicemia, urinary tract and gastrointestinal infections [110]. The Antibiotic Resistance Threats in the United States (2019) predict a total of 32,600 cases and 2700 fatalities, due to multidrug-resistant Pseudomonas aeruginosa [111]. Several studies investigated the level of $P$. aeruginosa contamination of commercially supplied packaged water around the world [112]. A study promoted by Sotler and collaborators [110] indicated the presence of P. aeruginosa in 33 of the 80 tested sachet water samples. The presence of this bacterium was reported in seven sachet water samples supplied in India [113]. In Abuja, Nigeria (2014), P. aeruginosa was isolated in $9.36 \%$ out of 100 brands of packaged water samples [114]. A similar investigation involving 120 sachet water samples collected from Owerri, Imo State, Nigeria (2011) revealed P. aeruginosa in $12 \%$ of the samples [115]. Furthermore, P. aeruginosa was also isolated from 8 of 38 routine post-pasteurization milk samples. In this strange case, all the pre-pasteurization milk samples were sterile. The P. aeruginosa contamination occurred during the water intake pipes in the milk bank and from cold water immersing milk bottles immediately after pasteurization [116].

P. aeruginosa growth was suppressed by an intermediate grade of C. barbata [50], C. tamariscifolia [60] and P. pavonica [60,88] (Phaeophyta), C. glomerata [50] (Chlorophyta) and L. papillosa [62], R. confervoides [60] and C. rubrum [88] (Rhodophyta). The extraction solvents most used are methanol and ethanol, as shown in Table 2. After a phytochemical screening, the alga species C. rubrum, S. vulgare, S. fusiforme and P.pavonia showed the presence of indoles, terpenes, acetogenins, phenols, fatty acids and volatile halogenated hydrocarbon. These alga were extracted and the solvent effect was investigated with diethyl ether, methanol, ethanol and chloroform, reporting the following results: S. vulgare presented results only with diethyl ether, S. fusiforme showed activity with diethyl ether $>$ methanol $>$ ethanol $>$ chloroform, P. pavonica showed activity with chloroform $>$ diethyl ether and ethanol $>$ methanol. C. rubrum showed activity with diethyl ether $>$ ethanol $>$ methanol and chloroform [88]. In another study focused on P. australis and S. polycystum from two different locations, best inhibition results were achieved with methanol and dichloromethane for P. australis and hexane for S. polycystum. Algae from different locations gave slightly different MICs [101]. 
Table 2. Antimicrobial effect of algae extracts in selected Gram-negative bacteria.

\begin{tabular}{|c|c|c|c|c|}
\hline Seaweed & Solvent & $\mathrm{MIC} / \mathrm{IC} 50 / \mathrm{MBC}(\mathrm{mg} / \mathrm{mL})$ & Agar Diffusion & Ref. \\
\hline \multicolumn{5}{|l|}{ Enterobacter spp. (E. cloacae) } \\
\hline G. ornata (R) & $\mathrm{H}_{2} \mathrm{O} / \mathrm{EtOH} \mathrm{f}$ & & $\operatorname{Re}$ & [64] \\
\hline Gracilaria sp. (R) & $\mathrm{MeOH}$ & & NI & [65] \\
\hline $\begin{array}{l}\text { H. cuneiformis (B)/S. ilicifolium }(\mathrm{B}) / S \text {. } \\
\text { incisifolium (B)/S. polycystum } \\
\text { (B)/Sargassum sp. (B)/T. conoides (B)/T. } \\
\text { decurrens (B)/T. ornata (B) }\end{array}$ & $\mathrm{MeOH}$ & & $\operatorname{Re}$ & [76] \\
\hline $\begin{array}{l}\text { L. complanata }(\mathrm{R}) / \text { Grateloupia sp. }(\mathrm{R}) / \mathrm{G} \text {. } \\
\text { corticata }(\mathrm{R}) / \text { Halymenia sp. }(\mathrm{R}) / \text { Spyridia } \mathrm{sp} \text {. } \\
(\mathrm{R}) / \text { Meta-mastophora sp. }(\mathrm{R}) / \text { Calloseris sp. } \\
(\mathrm{R}) / \mathrm{N} \text {. fraxinifolia }(\mathrm{R})\end{array}$ & $\mathrm{MeOH}$ & & $\operatorname{Re}$ & [77] \\
\hline U. reticulata $(\mathrm{G})$ & $\mathrm{MeOH}: \mathrm{CHCl}_{3}: \mathrm{H}_{2} \mathrm{O}$ & & $\mathrm{S}$ & [78] \\
\hline \multicolumn{5}{|l|}{ Proteus spp. (P. mirabilis; P. vulgaris) } \\
\hline L. papillosa (R) & DCM/DCM:MeOH/MeOH/ $\mathrm{H}_{2} \mathrm{O}$ & & $\operatorname{Re} / \operatorname{Re} / \operatorname{Re} / \operatorname{Re}$ & \\
\hline Sargassum binderi (B) & \multirow{4}{*}{$\mathrm{H}_{2} \mathrm{O} / \mathrm{EtOH}$} & & $\operatorname{Re} / \operatorname{Re}$ & \multirow{4}{*}{ [99] } \\
\hline Amphiroa sp. (R) & & & $\operatorname{Re} / \operatorname{Re}$ & \\
\hline T. conoides (B) & & & $\operatorname{Re} / \operatorname{Re}$ & \\
\hline Halimeda macroloba $(\mathrm{G})$ & & & $\mathrm{Re} / \mathrm{NI}$ & \\
\hline Galaxaura rugosa (R) & \multirow{2}{*}{$\mathrm{EtOH} / \mathrm{CHCL}_{3} \mathrm{f} / \mathrm{n}-\mathrm{BuOH} \mathrm{f} / \mathrm{H}_{2} \mathrm{O} \mathrm{f}$} & MIC (2.5/1.25/2.5/5) & $\mathrm{I} / \mathrm{S} / \mathrm{S} / \mathrm{I}$ & \multirow{2}{*}{ [97] } \\
\hline L. hawaiiana (R) & & MIC (-/2.5/0.625/10) & $\mathrm{NI} / \mathrm{I} / \mathrm{SI}$ & \\
\hline Polysiphonia hainanensis (R) & $\mathrm{EtOH} / \mathrm{AcO}$ & & $\operatorname{Re}$ & [29] \\
\hline Halidrys siliquosa (B) & $\mathrm{MeOH} / \mathrm{Hex} ; \mathrm{EtAc} \mathrm{f}$ & MIC (1.25) & & [117] \\
\hline T. conoides $(\mathrm{B})$ & \multirow{3}{*}{$\mathrm{MeOH} / \mathrm{CHCl}_{3} / \mathrm{EtOH} / \mathrm{DiEt} / \mathrm{PeEt} / \mathrm{EtAc} / \mathrm{AcO}$} & & $\operatorname{Re}$ & \multirow{3}{*}[101]{} \\
\hline P. gymnospora (B) & & & $\operatorname{Re}$ & \\
\hline S. tenerrimum (B) & & & $\operatorname{Re} / \mathrm{I} / \operatorname{Re} / \operatorname{Re} / \mathrm{I} / \mathrm{I} / \mathrm{I}$ & \\
\hline Padina tetrastromatica (B) & $\mathrm{MeOH}$ & & $\operatorname{Re}$ & [100] \\
\hline Sargassum muticum (B) & $\mathrm{AcO} / \mathrm{CHCl}_{3} / \mathrm{MeOH}$ & & $\mathrm{Re} / \operatorname{Re} / \operatorname{Re}$ & [98] \\
\hline
\end{tabular}


Table 2. Cont.

\begin{tabular}{|c|c|c|c|c|}
\hline Seaweed & Solvent & MIC/IC50/MBC (mg/mL) & Agar Diffusion & Ref. \\
\hline \multicolumn{5}{|c|}{ Salmonella spp. (S. choleraesuis; S. typhi; S. typhimurium; S. enterica; S. gallinarum) } \\
\hline L. brandenii $(\mathrm{R})$ & $\mathrm{MeOH}$ & & $\operatorname{Re}$ & [63] \\
\hline $\begin{array}{l}\text { L. complanata }(\mathrm{R}) / \text { Grateloupia sp. (R)/G. } \\
\text { corticata }(\mathrm{R}) / \text { Halymenia sp. (R)/Spyridia sp. } \\
(\mathrm{R}) / \text { Meta-mastophora sp. (R)/Calloseris sp. } \\
(\mathrm{R}) / \mathrm{N} \text {. fraxinifolia }(\mathrm{R})\end{array}$ & $\mathrm{MeOH}$ & & $\operatorname{Re}$ & [77] \\
\hline G. ornata (R) & $\mathrm{H}_{2} \mathrm{O} / \mathrm{EtOH} \mathrm{f}$ & & $\operatorname{Re}$ & [64] \\
\hline Cladophora socialis $(\mathrm{G})$ & \multirow{3}{*}{$\mathrm{AcO} / \mathrm{MeOH}$} & & S/I & \multirow{3}{*}{ [118] } \\
\hline Sargassum latifolium B (B) & & & $\mathrm{I} / \mathrm{I}$ & \\
\hline Sargassum platycarpum A (B) & & & $\operatorname{Re} / \operatorname{Re}$ & \\
\hline D. membranacea (B) & MeHO:DCM/EtOH f/AcO f & & $\operatorname{Re} / \mathrm{I} / \operatorname{Re}$ & [28] \\
\hline S. hytrix (B) & \multirow{8}{*}{ EtAc/MeOH } & & $\mathrm{Re} / \mathrm{NI}$ & \multirow{8}{*}[47]{} \\
\hline C. racemosa $(\mathrm{G})$ & & & $\mathrm{Re} / \mathrm{NI}$ & \\
\hline S. dentifolium (B) & & & $\mathrm{Re} / \mathrm{NI}$ & \\
\hline C. myrica $(\mathrm{G})$ & & & $\mathrm{NI} / \mathrm{Re}$ & \\
\hline P. gymnospora (B) & & & $\operatorname{Re} / \mathrm{I}$ & \\
\hline C. fragile $(\mathrm{G})$ & & & $\operatorname{Re}$ & \\
\hline A. fragilis (R) & & & $\mathrm{Re} / \mathrm{NI}$ & \\
\hline C. trinodis (B) & & & $\operatorname{Re} / \operatorname{Re}$ & \\
\hline $\begin{array}{c}\text { H. tuna }(\mathrm{G}) / D \text {. dichotoma var intricate } \\
(\mathrm{B}) / D \text {. indica }(\mathrm{B}) / \mathrm{M} \text {. afaqhusainii }(\mathrm{R}) / \mathrm{S} \text {. } \\
\text { lanceolatum (B) }\end{array}$ & $\mathrm{EtOH}: \mathrm{H}_{2} \mathrm{O}$ & & $\operatorname{Re} / \mathrm{I} / \mathrm{I} / \operatorname{Re} / \operatorname{Re}$ & [57] \\
\hline $\begin{array}{l}\text { C. glomerata }(\mathrm{G}) / \text { E. linza }(\mathrm{G}) / \text { U. rigida } \\
(\mathrm{G}) / \text { C. barbata }(\mathrm{B}) / \text { P. pavonica }(\mathrm{B}) / \mathrm{C} \text {. } \\
\text { ciliatum }(\mathrm{R}) / \text { C. officinalis }(\mathrm{R})\end{array}$ & $\mathrm{EtOH}$ & & $\mathrm{I} / \mathrm{Re} / \mathrm{I} / \operatorname{Re} / \mathrm{R} \mathrm{e} / \operatorname{Re} / \mathrm{I}$ & [50] \\
\hline D. membranacea (B) & $\mathrm{H}_{2} \mathrm{O} / \mathrm{CHCl}_{3 /} \mathrm{EtOAc}$ & & $\mathrm{NI} / \mathrm{NI} / \mathrm{NI}$ & [59] \\
\hline
\end{tabular}


Table 2. Cont

\begin{tabular}{|c|c|c|c|c|}
\hline Seaweed & Solvent & MIC/IC50/MBC (mg/mL) & Agar Diffusion & Ref. \\
\hline $\begin{array}{l}\text { S. lomentaria }(\mathrm{B}) / P . \text { pavonica }(\mathrm{B}) / \mathrm{C} \text {. } \\
\text { mediterranea }(\mathrm{B}) / \mathrm{H} \text {. musciformis }(\mathrm{R}) / \mathrm{S} \text {. } \\
\text { filamentosa }(\mathrm{R})\end{array}$ & $\mathrm{MeOH}$ & & I/NI/NI/NI/NI & [54] \\
\hline E. cava (B) & $\mathrm{EtOH} / \mathrm{n}-\mathrm{Hex} / \mathrm{DCM} / \mathrm{EtAc} / \mathrm{n}-\mathrm{BuOH} / \mathrm{H}_{2} \mathrm{O} / \mathrm{Ac}$ & $\begin{array}{c}\mathrm{MIC}(2 / \mathrm{NI} / \mathrm{NI} / 0.25 / 2 / \mathrm{NI} / 9.7 \times \\
\left.10^{-4}\right)\end{array}$ & & [109] \\
\hline C. barbata (B) & $\mathrm{EtOH}$ & & $\operatorname{Re}$ & [70] \\
\hline H. flagelliformis (R) & $\mathrm{MeOH}$ & & & \\
\hline C. myrica (B) & $\mathrm{MeOH}$ & & NI & [73] \\
\hline S. boveanum (B) & $\mathrm{H}_{2} \mathrm{O} / \mathrm{MeOH} / \mathrm{DCM}$ & & & \\
\hline S. japonica (B) & $\begin{array}{c}\text { Raw material/Raw material + } \\
\text { catalyst/De-oiled/de-oiled + catalyst }\end{array}$ & & $\mathrm{NI} / \mathrm{Re} / \mathrm{NI} / \mathrm{Re}$ & [44] \\
\hline H. esperi (R)/C. prolifera $(\mathrm{G})$ & $\mathrm{MeOH}$ & & NI & [75] \\
\hline $\begin{array}{l}\text { H. cuneiformis (B)/S. ilicifolium (B)/S. } \\
\text { polycystum }(\mathrm{B}) / \text { Sargassum sp. (B)/T. } \\
\text { conoides (B)/T. ornata (B) }\end{array}$ & $\mathrm{MeOH}$ & & $\operatorname{Re}$ & [76] \\
\hline \multicolumn{5}{|c|}{ Shigella sp. (reference strains: ATCC 9204 and 1457) } \\
\hline $\begin{array}{l}\text { H. cuneiformis }(\mathrm{B}) / \text { S. ilicifolium }(\mathrm{B}) / S \text {. } \\
\text { incisifolium }(\mathrm{B}) / \text { S. polycystum } \\
\text { (B)/Sargassum sp. (B)/T. conoides (B)/T. } \\
\text { decurrens }(\mathrm{B}) / T \text {. ornata (B) }\end{array}$ & MeOH:DCM & & $\operatorname{Re}$ & [76] \\
\hline S. muticum (B) & $\mathrm{CHCl}_{3 /} \mathrm{AcO} / \mathrm{MeOH}$ & & $\operatorname{Re}$ & [98] \\
\hline C. rubrum (R) & \multirow{4}{*}{$\mathrm{DiEt} / \mathrm{MeOH} / \mathrm{EtOH} / \mathrm{CHCl}_{3}$} & & $\operatorname{Re} / \operatorname{Re} / \mathrm{I} / \mathrm{Re}$ & \multirow{4}{*}{ [88] } \\
\hline S. vulgare (B) & & & $\mathrm{Re} / \mathrm{Re} / \mathrm{NI} / \mathrm{NI}$ & \\
\hline S. fusiforme (B) & & & $\operatorname{Re} / \mathrm{NI} / \operatorname{Re} / \operatorname{Re}$ & \\
\hline P. pavonia (B) & & & $\operatorname{Re} / \operatorname{Re} / \mathrm{S} / \operatorname{Re}$ & \\
\hline T. conoides $(\mathrm{B})$ & \multirow{3}{*}{$\mathrm{MeOH} / \mathrm{AcO} / \mathrm{PeEt} / \mathrm{EtOH} / \mathrm{EtAc} / \mathrm{CHCl}_{3} / \mathrm{DiEt}$} & & $\operatorname{Re}$ & \multirow{3}{*}{ [101] } \\
\hline P. gymnospora (B) & & & $\mathrm{I} / \mathrm{I} / \mathrm{Re} / \mathrm{Re} / \mathrm{I} / \mathrm{R}$ e/I & \\
\hline S. tenerrimum $(\mathrm{B})$ & & & $\mathrm{I} / \mathrm{Re} / \mathrm{I} / \mathrm{Re} / \mathrm{I} / \mathrm{R}$ e/I & \\
\hline
\end{tabular}


Table 2. Cont.

\begin{tabular}{|c|c|c|c|c|}
\hline Seaweed & Solvent & MIC/IC50/MBC (mg/mL) & Agar Diffusion & Ref. \\
\hline \multicolumn{5}{|c|}{ Pseudomonas aeruginosa (reference strains: ATCC 25619/27853/85327/9027; KCTC 1637; DSM 50071; MTCC 2453/424) } \\
\hline L. brandenii (R) & $\mathrm{MeOH}$ & & $\operatorname{Re}$ & [63] \\
\hline G. ornata (R) & $\mathrm{H}_{2} \mathrm{O} / \mathrm{EtOH} / \mathrm{EtOH}$ & & $\operatorname{Re}$ & [64] \\
\hline G. changii (R) & $\mathrm{MeOH}$ & MIC (6.25) & $\operatorname{Re}$ & [119] \\
\hline C. rupestris $(G)$ & $\mathrm{CHCl}_{3}: \mathrm{MeOH}$ & & $\operatorname{Re}$ & [120] \\
\hline C. trinodis $(B)$ & $\left(\mathrm{C}_{2} \mathrm{H}_{5}\right)_{2}: \mathrm{EtOH}: \mathrm{Hex}$ & MIC (6.6) & & [49] \\
\hline $\begin{array}{l}\text { C. myrica }(\mathrm{B}) / \text { C. trinodis }(\mathrm{B}) / \text { P. gymnospora } \\
(\mathrm{B}) / \text { S. dentifolium }(\mathrm{B}) / \text { S. hystrix }(\mathrm{B}) / \text { A. } \\
\text { fragilis }(\mathrm{R}) / \text { Caulerpa racemose }(\mathrm{G}) / \mathrm{C} \text {. fragile } \\
(\mathrm{G})\end{array}$ & $\mathrm{MeOH} / \mathrm{EtOH}$ & & $\operatorname{Re}$ & [47] \\
\hline P. australis (B) & \multirow{3}{*}{$\mathrm{MeOH} / \mathrm{DCM} / \mathrm{n}-\mathrm{Hex}$} & MIC (0.26/0.26/0.73) & & \multirow{3}{*}{ [48] } \\
\hline S.polycystum TK (B) & & MIC (0.73/0.21/0.10) & & \\
\hline S.polycystum CR (B) & & MIC (0.21/0.21/0.10) & & \\
\hline L. papillosa $(\mathrm{R})$ & DCM/DCM:MeOH/MeOH/ $\mathrm{H}_{2} \mathrm{O}$ & & $\operatorname{Re} / \mathrm{I} / \operatorname{Re} / \operatorname{Re}$ & [62] \\
\hline $\begin{array}{l}\text { U. flexuosa }(\mathrm{G}) / \text { P. antillarum }(\mathrm{B}) / P \text {. } \\
\text { boergeseni }(\mathrm{B})\end{array}$ & $\mathrm{EtAc} / \mathrm{MeOH}$ & & NI & [66] \\
\hline G. doryphora (R) & $\mathrm{EtOH} / \mathrm{MeOH} / \mathrm{EtAc}$ & & $\operatorname{Re}$ & [68] \\
\hline Eisenia bicyclis (B) & $\mathrm{MeOH} / \mathrm{Hex} / \mathrm{DCM} / \mathrm{EtAc} / \mathrm{BuOH}$ & & NI & [121] \\
\hline $\begin{array}{l}\text { H. tuna }(\mathrm{G}) / \text { D. dichotoma var intricate } \\
\text { (B)/D. indica (B)/M. afaqhusainii (R)/S. } \\
\text { lanceolatum (B) }\end{array}$ & $\mathrm{EtOH} / \mathrm{H}_{2} \mathrm{O}$ & & $\operatorname{Re}$ & [57] \\
\hline $\begin{array}{c}\text { C. glomerata }(\mathrm{G}) / \text { E. linza }(\mathrm{G}) / \mathrm{U} \text {. rigida } \\
(\mathrm{G}) / \text { C. barbata }(\mathrm{B}) / \text { C. ciliatum }(\mathrm{R}) / C \text {. } \\
\text { officinalis }(\mathrm{R})\end{array}$ & $\mathrm{EtOH}$ & & $\mathrm{I} / \operatorname{Re} / \operatorname{Re} / \mathrm{e} \operatorname{Re} / \operatorname{Re} / \operatorname{Re}$ & [50] \\
\hline D. membranacea (B) & $\mathrm{H}_{2} \mathrm{O} / \mathrm{CHCl}_{3 /} \mathrm{EtAc}$ & & $\mathrm{NI} / \mathrm{NI} / \mathrm{NI}$ & [59] \\
\hline
\end{tabular}


Table 2. Cont.

\begin{tabular}{|c|c|c|c|c|}
\hline Seaweed & Solvent & $\mathrm{MIC} / \mathrm{IC} 50 / \mathrm{MBC}(\mathrm{mg} / \mathrm{mL})$ & Agar Diffusion & Ref. \\
\hline $\begin{array}{c}\text { C. linum }(\mathrm{G}) / \text { C. rupestris }(\mathrm{G}) / \text { F. serratus } \\
(\mathrm{B}) / \text { F. vesiculosus }(\mathrm{B}) / H \text {. tomentosus }(\mathrm{B}) / \mathrm{S} \text {. } \\
\text { latissima }(\mathrm{B}) / B \text {. hamifera }(\mathrm{R}) / \text { C. corymbosum } \\
(\mathrm{R}) / \text { C. tenuicorn }(\mathrm{R}) / \text { Ceramium virgatum } \\
(\mathrm{R}) / D \text {. baillouviana }(\mathrm{R}) / D \text {. sanguinea }(\mathrm{R}) / D \text {. } \\
\text { contorta }(\mathrm{R}) / P \text {. elongata }(\mathrm{R}) / P \text {. nigra }(\mathrm{R}) / \mathrm{R} \text {. } \\
\text { confervoides }(\mathrm{R})\end{array}$ & $\mathrm{DCM}$ & & NI & [69] \\
\hline B. bifurcata (B) & $\mathrm{MeOH} / \mathrm{DCM}$ & & $\operatorname{Re} / \operatorname{Re}$ & [53] \\
\hline T. conoides (B) & n-Hex/ $\mathrm{C}_{6} \mathrm{H}_{12 /} \mathrm{MeOH} / \mathrm{EtOH}: \mathrm{H}_{2} \mathrm{O}$ & & $\mathrm{NI} / \mathrm{R} \mathrm{e} / \mathrm{Re} / \mathrm{NI}$ & [74] \\
\hline $\begin{array}{l}\text { C. tamariscifolia }(\mathrm{B}) / \text { P. pavonica }(\mathrm{B}) / \mathrm{R} \text {. } \\
\text { confervoides }(\mathrm{R}) / \text { U. lactuca }(\mathrm{G})\end{array}$ & $\mathrm{MeOH}$ & & $\mathrm{I} / \mathrm{I} / \mathrm{I} / \operatorname{Re}$ & [60] \\
\hline E. prolifera $(\mathrm{G})$ & DiEt & $\operatorname{MIC}\left(1.25 \times 10^{-3}\right)$ & & [58] \\
\hline S. portieriatum (B) & $\mathrm{MeOH}$ & $\operatorname{MIC}\left(7.5 \times 10^{-4}\right)$ & & \\
\hline H. esperi (R) & \multirow{2}{*}{$\mathrm{MeOH}$} & & NI & \multirow{2}{*}{ [75] } \\
\hline C. prolifera $(\mathrm{G})$ & & & I & \\
\hline $\begin{array}{l}\text { H. cuneiformis }(\mathrm{B}) / \text { S. ilicifolium }(\mathrm{B}) / \mathrm{T} \text {. } \\
\text { ornata }(\mathrm{B})\end{array}$ & $\mathrm{MeOH}$ & & $\mathrm{NI} / \operatorname{Re} / \operatorname{Re}$ & [76] \\
\hline H. elongata (B) & $\mathrm{H}_{2} \mathrm{O} / \mathrm{MeOH}$ & & $\operatorname{Re}$ & [30] \\
\hline U. rigida $(\mathrm{G})$ & $\begin{array}{c}\text { EtOH: } \mathrm{H}_{2} \mathrm{O} / \mathrm{Hex} / \mathrm{CHCl}_{3} \mathrm{f} / \mathrm{EtOAc} \mathrm{f} / \mathrm{ButOH} \\
\mathrm{f} / \mathrm{H}_{2} \mathrm{O}\end{array}$ & & $\mathrm{Re} / \mathrm{NI} / \mathrm{Re} / \mathrm{Re} / \mathrm{NI} / \mathrm{NI}$ & [45] \\
\hline S. vulgare (B) & \multirow{4}{*}{$\mathrm{DIEt} / \mathrm{MeOH} / \mathrm{EtOH} / \mathrm{CHCl}_{3}$} & & $\mathrm{Re} / \mathrm{NI} / \mathrm{NI} / \mathrm{NI}$ & \multirow{4}{*}[88]{} \\
\hline S. fusiforme (B) & & & $\mathrm{S} / \mathrm{Re} / \mathrm{Re} / \mathrm{NI}$ & \\
\hline P. pavonia (B) & & & $\mathrm{I} / \mathrm{NI} / \mathrm{I} / \mathrm{I}$ & \\
\hline C. rubrum (R) & & & $\mathrm{I} / \mathrm{NI} / \mathrm{I} / \mathrm{NI}$ & \\
\hline
\end{tabular}

Seaweed information: (G)—green algae (Chlorophytes); (R)—red algae (Rhodophytes); (B)—brown algae (Phaeophyta); nd—not determined. Solvents: AcO-acetone;

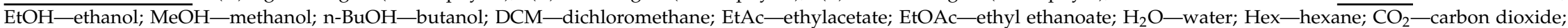
DMSO-dimethilsulfoxide; $\mathrm{CHCl}_{3}$-chloroform; BuOH—butanol; PeEt—petroleum ether; DIEt—diethyl ether; PolySA-polysaccharides agar; $\mathrm{f}-$ fraction. Agar diffusion: Re-resistant $\leq 14 \mathrm{~mm}$; I-intermediate 15-19 $\mathrm{mm}$; S-susceptible $\geq 20 \mathrm{~mm}$ [32]; NI—no inhibition. 


\subsection{Gram Positive}

\subsubsection{Staphylococcus aureus}

Staphylococcus aureus is a facultative anaerobic coccus, which is non-motile and catalase and coagulase positive. Among the predominant foodborne bacteria described as human pathogens, S. aureus is the foremost cause of gastroenteritis [122], but contamination can be readily avoided by heat treatment of food. Researchers assessed the prevalence of $S$. aureus and its enterotoxins in raw pork and smoked hams produced from this meat [123]. In total, the pathogen was found in $25.9 \%$ of the 135 samples by culture techniques and $S$. aureus genes were detected by polymerase chain reaction (PCR) in $51.1 \%$ of the examined samples. From $11.1 \%$ of the ready-for-sale smoked hams, S. aureus was isolated and $35.6 \%$ of this product reacted positively in the PCR. Therefore, concerning the consumers' health, these results have to be critically evaluated [123]. Although S. aureus can be easily found on the skin and hair of warm-blooded animals, up to $30-50 \%$ of the human population are carriers of these bacteria [122]. This bacterium is notorious for its resistance to multiple antibiotics [124].

Concerning the antibacterial activity from bioactive compounds from macroalgae on S. aureus, several studies have been performed within Chlorophyta, Rhodophyta and Phaeophyta phylum.

Usually, different extraction solvents are used to obtain bioactive compounds (alkaloids, polyketides, cyclic peptide, polysaccharide, phlorotannins, diterpenoids, sterols, quinones, lipids and glycerols) [29]: methanol (most used), ethanol, acetone, dichloromethane, hexane, diethyl ether, ethyl acetate, dimethilsulfoxide (DMSO), chloroform and solvent mixtures. The strain most evaluated was ATCC 25923 but also activity against methicillin-resistant S. aureus (MRSA) was tested, as shown in Table 3. Acetone extracts of L. obtusa, C. elongatum and C. multifida obtained the lowest MIC at $0.625 \mu \mathrm{g} / \mathrm{mL}$ [125]. Among Chlorophyta, the genus Ulva spp. was reported in most of the studies against $S$. aureus $[45,50,66,80,125-128]$. Some studies compared solvent extraction performance for extraction of bioactive compounds: for C. socialis (MeOH > acetone) [118], U. flexuosa (ethylacetate > $\mathrm{MeOH}$ ) and for $U$. rigida (ethanol $>\mathrm{Hex}>\mathrm{CHCl}_{3}$ ). Regarding Rhodophyta, the genus with the most described studies is Laurencia spp. [52,62,77,125,127]. Relating to Phaeophytha phylum, Sargassum spp. was the most studied genus $[41,57,73,76,99,118,129,130]$. Comparing solvent performance for bioactive compounds extraction, the following results were obtained: ethanol or acetone $>$ crude extract or $\mathrm{MeOH} / \mathrm{DCM}$ for D. membranacea [28], EtOAc $>\mathrm{EtOH}>$ hexane, DCM, n-BuOH, water $\left(\mathrm{H}_{2} \mathrm{O}\right)$ for $E$. cava [109]; EtOAc $>$ DCM, BuOH $>$ hexane $>\mathrm{MeOH}$ for E. bicyclis [121]; $\mathrm{H}_{2} \mathrm{O}>\mathrm{MeOH}$, acetone or ethyl acetate for F. serratus [131]; ethanol $>$ hexane $>\mathrm{H}_{2} \mathrm{O}$ for $H$. elongata [21]; ethylacetate $>\mathrm{MeOH}$ for P. antillarum and P. boergeseni [66]; $\mathrm{MeOH}>$ acetone for S. platycarpum and for S. latifolium [118]. It can be concluded that there is not a common solvent to extract compounds with antibacterial activity against $S$. aureus. 
Table 3. Antimicrobial effect of alga extracts on Staphylococcus aureus (reference strains: ATCC 25923/2940/11632/6538/6462/333591; TISTR517; CMCC(B)26003; BAA-42; PTCC1112; RCM 010027).

\begin{tabular}{|c|c|c|c|c|}
\hline Seaweed & Solvent & MIC/IC50/MBC (mg/mL) & Agar Diffusion & Ref. \\
\hline C. socialis $(\mathrm{G})$ & \multirow{3}{*}{$\mathrm{AcO} / \mathrm{MeOH}$} & & $\mathrm{I} / \mathrm{S}$ & \multirow{3}{*}[118]{} \\
\hline S. latifolium (B) & & & $\mathrm{I} / \mathrm{S}$ & \\
\hline S. platycarpum (B) & & & $\operatorname{Re} / \mathrm{I}$ & \\
\hline $\begin{array}{l}\text { C. elongatum (G)/L. obtusa (R)/C. } \\
\text { multifida (B) }\end{array}$ & $\mathrm{AcO}$ & $\operatorname{MIC}\left(0.63 \times 10^{3}\right)$ & & [125] \\
\hline $\begin{array}{l}\text { H. macroloba (G)/S. binderi } \\
\text { (B)/Amphiroa sp. (B)/T. conoides } \\
\text { (B) }\end{array}$ & $\mathrm{AcO}$ & & $\operatorname{Re}$ & [99] \\
\hline $\begin{array}{l}\text { U. lactuca }(\mathrm{G}) / \text { C. fragile }(\mathrm{G}) / \mathrm{L} . \\
\text { johnstonii }(\mathrm{R}) / \text { Gymnogongrus } \\
\text { martinensis }(\mathrm{R}) / \text { D. flabellata } \\
\text { (B)/Padina concrescens }(\mathrm{B})\end{array}$ & AcO:MeOH & & $\mathrm{Re} / \mathrm{NI} / \mathrm{I} / \mathrm{NI} / \mathrm{NI} / \mathrm{NI}$ & [127] \\
\hline \multirow{3}{*}{$\begin{array}{l}\text { U. flexuosa }(\mathrm{G}) / \text { P. antillarum (B)/P. } \\
\text { boergeseni (B) }\end{array}$} & \multirow{3}{*}{$\mathrm{MeOH} / \mathrm{EtAc}$} & MIC (3.75/1.87) & $\mathrm{I} / \mathrm{S}$ & \multirow{3}{*}{ [66] } \\
\hline & & MIC (7.5/3.75) & $\mathrm{I} / \mathrm{S}$ & \\
\hline & & MIC (15/3.75) & $\operatorname{Re} / \mathrm{I}$ & \\
\hline \multirow{2}{*}{ U. rigida $(\mathrm{G})$} & $\mathrm{DCM}$ & & \multirow{2}{*}{$\operatorname{Re}$} & \multirow{2}{*}{ [80] } \\
\hline & DCM:MeOH & & & \\
\hline $\begin{array}{l}\text { H. tuna }(\mathrm{G}) / \text { D. dichotoma var } \\
\text { intricata }(\mathrm{B}) / D \text {. indica }(\mathrm{B}) / S \text {. } \\
\text { lanceolatum }(\mathrm{B}) / \text { D. dichotoma var } \\
\text { intricata }(\mathrm{B}) / \text { M. afaqhusainii }(\mathrm{R})\end{array}$ & $\mathrm{EtOH}$ & & $\operatorname{Re}$ & [57] \\
\hline $\begin{array}{c}\text { C. glomerata }(\mathrm{G}) / \text { E. linza }(\mathrm{G}) / \mathrm{U} \text {. } \\
\text { rigida }(\mathrm{G}) / \mathrm{C} \text {. ciliatum }(\mathrm{R}) / \mathrm{C} \text {. } \\
\text { barbata }(\mathrm{B}) / P \text {. pavonica }(\mathrm{B}) / \mathrm{C} \text {. } \\
\text { officinalis }(\mathrm{B})\end{array}$ & $\mathrm{EtOH}$ & $\begin{array}{c}\text { MIC } \\
(>1.25 />2.5 />2.5 />10 />1.25 />1.25 />5)\end{array}$ & $\mathrm{I} / \operatorname{Re} / \mathrm{I} / \operatorname{Re} / \mathrm{I} / \mathrm{I} / \operatorname{Re}$ & [50] \\
\hline $\begin{array}{l}\text { C. nodosa }(\mathrm{G}) / \text { H. tuna }(\mathrm{G}) / \mathrm{C} \text {. } \\
\text { barbata }(\mathrm{G}) / \text { C. bursa }(\mathrm{G})\end{array}$ & $\mathrm{MeOH}$ & & $\operatorname{Re}$ & [51] \\
\hline
\end{tabular}


Table 3. Cont

\begin{tabular}{|c|c|c|c|c|}
\hline Seaweed & Solvent & MIC/IC50/MBC (mg/mL) & Agar Diffusion & Ref. \\
\hline Oedogonium sp. (G) & \multirow{4}{*}{$\mathrm{MeOH} / \mathrm{EtOH}$} & & NI & \multirow{4}{*}{ [72] } \\
\hline Stigeoclonium sp. (G) & & & $\operatorname{Re} / \mathrm{I}$ & \\
\hline Ulothrix sp. (G) & & & $\operatorname{Re}$ & \\
\hline Nitzschia sp. (G) & & & $\operatorname{Re} / \mathrm{I}$ & \\
\hline E. prolifera $(\mathrm{G})$ & EtAc & $\operatorname{MIC}\left(1.0 \times 10^{3}\right)$ & & \multirow{2}{*}{ [81] } \\
\hline P. pavonica (B) & PeEt & $\operatorname{MIC}\left(1.25 \times 10^{3}\right)$ & & \\
\hline C. prolifera $(\mathrm{G}) /$ H. esperi $(\mathrm{R})$ & $\mathrm{MeOH}$ & MIC (0.6/0.5) & I & [75] \\
\hline U. reticulata $(\mathrm{G})$ & $\mathrm{H}_{2} \mathrm{O}$ & & S & [128] \\
\hline U. rigida $(\mathrm{G})$ & $\mathrm{Hex} / \mathrm{CHCl}_{3} / \mathrm{EtOAc} / \mathrm{ButOH} / \mathrm{H}_{2} \mathrm{O} / \mathrm{Hex} / \mathrm{CHCl}_{3} / \mathrm{EtOAc} / \mathrm{ButOH} / \mathrm{H}_{2} \mathrm{O}$ & & \multicolumn{2}{|c|}{$\mathrm{NI} / \mathrm{NI} / \operatorname{Re} / \operatorname{Re} / \operatorname{Re} / \mathrm{I} / \mathrm{I} / \mathrm{Re} / \mathrm{NI} / \mathrm{NI}[45]$} \\
\hline $\begin{array}{l}\text { Ulva fasciata }(\mathrm{G}) / \text { Chaetomorpha } \\
\text { antennina }(\mathrm{G}) / \text { Caula anthus } \\
\text { okamurai }(\mathrm{R}) / \text { Ahnfeltiopsis } \\
\text { masudai }(\mathrm{R}) / \text { P. hainanensis } \\
\text { (R)/Sargassum hemiphyllum } \\
\text { (R)/Sargassum vachellianum } \\
\text { (R)/Pachydictyon coriaceum (B) }\end{array}$ & $\mathrm{EtOH}$ & & $\operatorname{Re} / \operatorname{Re} / \operatorname{Re} / \operatorname{Re} / \operatorname{Re} / \mathrm{I} / \mathrm{I} / \operatorname{Re}$ & [29] \\
\hline L. brandenii (R) & $\mathrm{CHCl}_{3} / \mathrm{MeOH}$ & & I & [63] \\
\hline G. ornata (R) & $\mathrm{MeOH}$ & & $\operatorname{Re}$ & [64] \\
\hline Gracilaria sp. (R) & PolySA/MeOH & & NI & [65] \\
\hline L. papillosa (R) & $\mathrm{EtOH} / \mathrm{MOH} / \mathrm{AcO}$ & & $\operatorname{Re}$ & [62] \\
\hline G. corticata (R)/G. edulis (R) & $\mathrm{MeOH} / \mathrm{DMSO}$ & & $\operatorname{Re}$ & [61] \\
\hline $\begin{array}{l}\text { H. musciformis (R)/S. filamentosa } \\
\text { (R)/S. lomentaria (B)/P. pavonica } \\
\text { (B)/C. mediterranea (B) }\end{array}$ & $\mathrm{MeOH}$ & & $\operatorname{Re}$ & [54] \\
\hline $\begin{array}{l}\text { A. fragilis }(\mathrm{R}) / \text { L. papillosa }(\mathrm{R}) / \mathrm{S} \text {. } \\
\text { cinereum }(\mathrm{R}) / \text { C. myrica }(\mathrm{B}) / \mathrm{H} \text {. } \\
\text { cuneiformis }(\mathrm{B}) / \text { T. turbinata }(\mathrm{B})\end{array}$ & DMSO & & $\operatorname{Re}$ & [52] \\
\hline
\end{tabular}


Table 3. Cont

\begin{tabular}{|c|c|c|c|c|}
\hline Seaweed & Solvent & MIC/IC50/MBC (mg/mL) & Agar Diffusion & Ref. \\
\hline H. flagelliformis (R) & \multirow{3}{*}{$\mathrm{DCM} / \mathrm{MeOH}$} & & $\operatorname{Re}$ & \multirow{3}{*}{ [73] } \\
\hline C. myrica (B) & & & $\mathrm{NI}$ & \\
\hline S. boveanum (B) & & & $\mathrm{NI}$ & \\
\hline $\begin{array}{l}\text { R. confervoides }(\mathrm{R}) / \mathrm{U} \text {. lactuca } \\
(\mathrm{G}) / \text { C. tamariscifolia }(\mathrm{B}) / P \text {. } \\
\text { pavonica }(\mathrm{B})\end{array}$ & $\mathrm{MeOH}$ & & I & [60] \\
\hline $\begin{array}{l}\text { L. complanata (R)/Grateloupia sp. } \\
(\mathrm{R}) / \text { G. corticata }(\mathrm{R}) / \text { Halymenia sp. } \\
(\mathrm{R}) / \text { Spyridia sp. } \\
(\mathrm{R}) / \text { Metamastophora sp. } \\
(\mathrm{R}) / \text { Calloseris sp. }(\mathrm{R}) / \mathrm{N} . \\
\text { fraxinifolia }(\mathrm{R})\end{array}$ & $\mathrm{MeOH}$ & & $\operatorname{Re}$ & [77] \\
\hline P. gymnospora (B) & $\mathrm{H}_{2} \mathrm{O}$ & $\operatorname{MIC}\left(500 \times 10^{3}\right)$ & & {$[132]$} \\
\hline Sargassum oligocystum (B) & $\mathrm{Hot} \mathrm{H}_{2} \mathrm{O}$ & MIC (3.18) & & [129] \\
\hline E. bicyclis (B) & $\mathrm{MeOH} / \mathrm{Hex} / \mathrm{DCM} / \mathrm{EtAc} / \mathrm{BuOH}$ & $\begin{array}{c}\text { MIC }\left(1.02 / 256 / 512 / 128 \times 10^{3} / 512 \times\right. \\
\left.10^{3}\right)\end{array}$ & $\operatorname{Re}$ & {$[121]$} \\
\hline C. trinodis (B) & DIEt:EtOH:Hex & MIC (1.031) & & [49] \\
\hline S. vulgare (B) & EtAc & & $\operatorname{Re}$ & {$[130]$} \\
\hline D. membranacea (B) & $\mathrm{H}_{2} \mathrm{O} / \mathrm{DCM} / \mathrm{EtAc}$ & & $\operatorname{Re}$ & [59] \\
\hline E. cava (B) & $\mathrm{EtOH} / \mathrm{n}-\mathrm{Hex} / \mathrm{DCM} / \mathrm{EtAc} / \mathrm{n}-\mathrm{BuOH} / \mathrm{H}_{2} \mathrm{O}$ & $\begin{array}{c}\text { MIC }\left(500 \times 10^{3} / \mathrm{nd} / \mathrm{nd} / 250 \times\right. \\
\left.10^{3} / \mathrm{nd} / \mathrm{nd}\right)\end{array}$ & & [109] \\
\hline C. barbata (B) & $\mathrm{EtOH}$ & & $\operatorname{Re}$ & [70] \\
\hline B. bifurcata $(\mathrm{B})$ & $\mathrm{MeOH} / \mathrm{DCM}$ & & $\mathrm{Re} / \mathrm{NI}$ & [53] \\
\hline $\begin{array}{c}\text { D. dichotoma (B)/P. pavonia (B)/S. } \\
\text { vulgare (B) }\end{array}$ & $\mathrm{AcO}$ & MIC (1.25/1.25/2.5) & & [41] \\
\hline F. vesiculosus (B) & $\mathrm{EtOH}$ & $\mathrm{IC}_{50}:(1.25)$ & & [43] \\
\hline S. japonica (B) & $\mathrm{CO}_{2}$ & MIC (3) & & [44] \\
\hline
\end{tabular}


Table 3. Cont.

\begin{tabular}{|c|c|c|c|c|}
\hline Seaweed & Solvent & $\mathrm{MIC} / \mathrm{IC} 50 / \mathrm{MBC}(\mathrm{mg} / \mathrm{mL})$ & Agar Diffusion & Ref. \\
\hline D. membranacea (B) & $\mathrm{MeOH}: \mathrm{DCM} / \mathrm{EtOH} / \mathrm{AcO}$ & & $\mathrm{Re} / \mathrm{S} / \mathrm{S}$ & [28] \\
\hline H. elongata (B) & $\mathrm{Hex} / \mathrm{EtOH} / \mathrm{H}_{2} \mathrm{O}$ & MBC (8.25/7.00/13.0) & & [21] \\
\hline $\begin{array}{c}\text { H. cuneiformis (B)/S. ilicifolium } \\
\text { (B)/S. incisifolium (B)/Sargassum } \\
\text { sp. (B)/T. conoides (B)/T. decurrens } \\
\text { (B)/T. ornata (B) }\end{array}$ & $\mathrm{MeOH}$ & & $\operatorname{Re}$ & [76] \\
\hline F. serratus (B)/F. vesiculosus (B) & $\mathrm{H}_{2} \mathrm{O} / \mathrm{MeOH} / \mathrm{AcO} / \mathrm{EtAc}$ & & I & [131] \\
\hline
\end{tabular}




\subsubsection{Listeria monocytogenes}

Listeria monocytogenes is a non-spore-forming, facultative anaerobic foodborne pathogen that can reproduce at refrigeration temperatures as well as at acidic $\mathrm{pH}$ and high salt concentrations and shows high resistance to disinfectants. In the food industry, this pathogen can colonize the environment, equipment and utensils and form biofilms, where it can remain for several months or even years causing cross-contamination [133]. Listeriosis is a serious infection usually caused by eating food contaminated with the bacterium L. monocytogenes. In major cases of young people and adults, it provokes a central nervous system infection and/or generalized infection. However, it is particularly dangerous in pregnancy, due to the ability of this bacterium to cause intrauterine infection and severe systemic infections in the unborn or newly delivered infant. Listeriosis can also arise in any gestation phase and affects children under one month old [134].

The prevalence of Listeria sp. and specifically L. monocytogenes in 650 ready-to-eat products (RTE) and 263 ingredients of salads and desserts was evaluated in Poland [135]. Unfortunately, 18\% of the samples were contaminated with Listeria sp. and $13.5 \%$ with L. monocytogenes. [135]. Moreover, 124 RTE samples composed of ground beef and chicken meat were also assessed and $81.5 \%$ of the samples presented contamination with Listeria sp., $35.5 \%$ with Listeria innocua and $26.6 \%$ with L. monocytogenes. In the years of 1998-2014, a total 58 outbreaks of listeriosis were reported in the US, $30 \%$ of those cases were associated with soft cheese [136].

Brown alga extracts obtained with different solvents were tested against Listeria sp. growth. Experiments made with D. membranacea (extracted in $\mathrm{H}_{2} \mathrm{O}$ ) [59] and C. glomerata (extracted in EtOH) [50] led to inhibition halos of intermediate diameter (Table 4). On the other hand, the brown alga species Laminaria digitata, Laminaria saccharina, H. elongata and the red algae Palmaria palmata, a seaweed rich in tannins, phenolics and flavonoids, were able to suppress the Listeria sp. growth ranging from very strong to moderate intensity [137].

\subsubsection{Enterococus spp.}

Enterococcus spp. can be found in several reservoirs, namely soil, water, vegetable products, meats, fermented and cooked meat and dairy products. Furthermore, these microorganisms are present as common microbiota in the intestine of humans, mammals and other animal gastrointestinal tracts. Enterococcus spp. is a facultative anaerobe bacterium proficient to subsist at high temperatures and in a broad range of $\mathrm{pH}$ conditions [138]. However, due to its adaptability, Enterococcus can endure several niches, serving as a parameter in sanitary quality of food [139].

Epidemiology studies referred to Enterococcus spp. as a human pathogen liable for $5 \%$ to $10 \%$ of endocarditis infections [140], in addition to being responsible for other illnesses such as urinary tract, central nervous system and pelvic infections. Enterococcus resistant to the glycopeptide antibiotics, vancomycin and teicoplanin, are also emerging [111,141]. Algae extracts were tested mainly against two different Enterococcus species, E. faecalis and E. faecium. The growth of E. faecium was successfully constrained by the crude extract of $D$. membranacea and by the ethanolic and acetone crude fractions containing the following bioactive molecules: catechin, polyphenols, tannin and phlorotannins [28], whereas the chloroform fraction of the green alga $U$. rigida crude extract (rich in phenolics, diterpenoids, terpenes, sterols, fatty acids), proved its efficiency against the E. faecalis [45]. Some authors have also studied the seasonal influence of the $U$. rigida performance as an antimicrobial agent. They showed that the algae harvesting season has an effect on the performance of the extract [142].

\subsubsection{Bacillus cereus}

Bacillus cereus is a facultative aerobic spore-forming bacterium able to thrive in temperatures ranging from $7{ }^{\circ} \mathrm{C}$ to $30^{\circ} \mathrm{C}$ [143]. This bacterium is a well-known foodborne pathogen, frequently widespread in soil and growing plants, nevertheless it is also able to grow in the intestinal tract of insects and mammals. From these habitats, it is easily spread to foods, where it may cause an emetic or 
a diarrheal type of food-associated illness that is becoming progressively more prevalent in the food industry [144,145]. An extensive study on the prevalence of $B$. cereus isolated from dairy products in China was performed [146]. B. cereus strains were isolated from 500 dairy product samples and a contamination rate of $11 \%$ was achieved. Raw milk presented the highest $B$. cereus contamination rate $(26 \%)$ followed by pasteurized milk samples $(12 \%)$, cheese samples $(10 \%)$, ultra-pasteurized milk samples (8\%) and powder infant formula samples (7\%) [146]. In another survey, approximately three hundred $B$. cereus strains were isolated from vegetables in different cities in China and were detected in $50 \%$ of the 419 samples [147].

Even though B. cereus spreads by spore formation, there is not enough evidence of the relationship between toxin production and biofilm formation. Moreover, there is also scarce evidence of the role of sporulation in the toxin production of B. cereus. As a foodborne pathogen, toxin production is important, and any sporulation and biofilm formation are expected to worsen the risk of food poisoning [145]. In the pursuit of alga extracts with an antagonistic effect on B. cereus growth, a large number of algae was tested by the agar diffusion method, but most were included in the resistant category, as shown in Table 4. Nevertheless, cholesterol and oxygenated cholesterol derivatives, brominated indoles 1-3 along with sesquiterpene, debilone, and mixture of fatty acid esters detected [77] in P. coriaceum, C. antennina and Codium cylindricum acetone extract [29] and in L. complanata (R) methanolic extract, presented encouraging results with the diameter of the inhibition growth zone higher than $15 \mathrm{~mm}$. The green seaweed E. linza was extracted to obtain essential oils and tested against two different B. cereus strains: ATCC 10876 and 13061. However, the MIC results were quite different, inferring that the strain of the microbe tested played an important role in the final experiment outcome [148]. It was also noticed that the inhibition capacity of the extracted compounds from $P$. coriaceum collected in different locations also corresponded to slightly different results [29].

\subsubsection{Streptococcus spp.}

The genus Streptococcus consists of 104 recognized species, both commensal and pathogenic. Major pathogenic species for human and animals are S. pneumoniae (middle ear infections, meningitis and pneumonia in children and pneumonia with sepsis in adults or immunocompromised persons), S. pyogenes (scarlet fever, rheumatic fever), S. agalactiae (neonatal pneumonia in human, mastitis in animals), S. dysgalactiae subsp. dysgalactiae [149] (mastitis), S. dysgalactiae subsp. equisimilis (strangles in horses, joint ill, mastitis in animals), S. gordonii (infective endocarditis in human), S. parasanguinis (infective endocarditis in human), S. sanguinis (infective endocarditis in human) and S. mutans (dental caries). An epidemiological investigation performed by Yamaguchi et al., [150] revealed the presence of $S$. dysgalactiae in a broccoli salad. Unfortunately, this contaminated food provoked a foodborne outbreak and 140 patients presented primary symptoms of sore throat and fever [150]. Bovine mastitis is the most frequent disease worldwide in dairy herds, causing high economic losses for producers and the industry, as well as having implications for public health due to the zoonotic potential of some agents involved in its etiology and the increased risk of antimicrobial residues in milk and its derivatives [151].

The prevalence of S. agalactiae in 306 dairy herds from Campo das Vertentes region, located in the south of Minas Gerais state, Brazil, was evaluated. The study involved approximately $3.4 \times 10^{4}$ dairy cows and covered an area of approximately $1.2 \times 10^{4} \mathrm{~km}^{2}$ and indicated that $67 \%$ of the samples were contaminated with S. agalactiae [151]. S. agalactiae was also found in bulk milk from Prince Edward Island dairy farms [152]. Furthermore, a high prevalence of $S$. infantarius was found in fermented milk of East and West Africa [153] The most relevant results related with the antimicrobial capacity of algae against Streptococcus spp. are summarized in Table 4. Among the algae tested against S. pneumoniae, the best inhibition results were obtained with S. incisifolium, Sargassum sp. [76]—an algae containing sterol and terpens-and L. complanata extracts [77]. The extracts of G. rugosa and L. hawaiiana were active against S. mutans [97]. The extract of G. rugosa also negatively affected the growth of S. pyogenes. Several seaweeds extracts (T. conoides, P. gymnospora and S. tenerrimum) obtained with different solvents 
were tested against Streptococcus sp. and the best inhibition results were obtained with acetone, ethyl acetate and methanol, respectively [101]. Laminaria japonica ethanolic extracts were used to restrain the growth of oral related bacteria (S. gordonii, S. mitis, S. mutans, S. oralis, S. sanguinis and S. sobrinus). MICs were between 60 to $250 \mu \mathrm{g} / \mathrm{mL}$, indicating that it is reasonable to assume future applications in dental care products.

Table 4. Antimicrobial effect of alga extracts in selected Gram-positive bacteria.

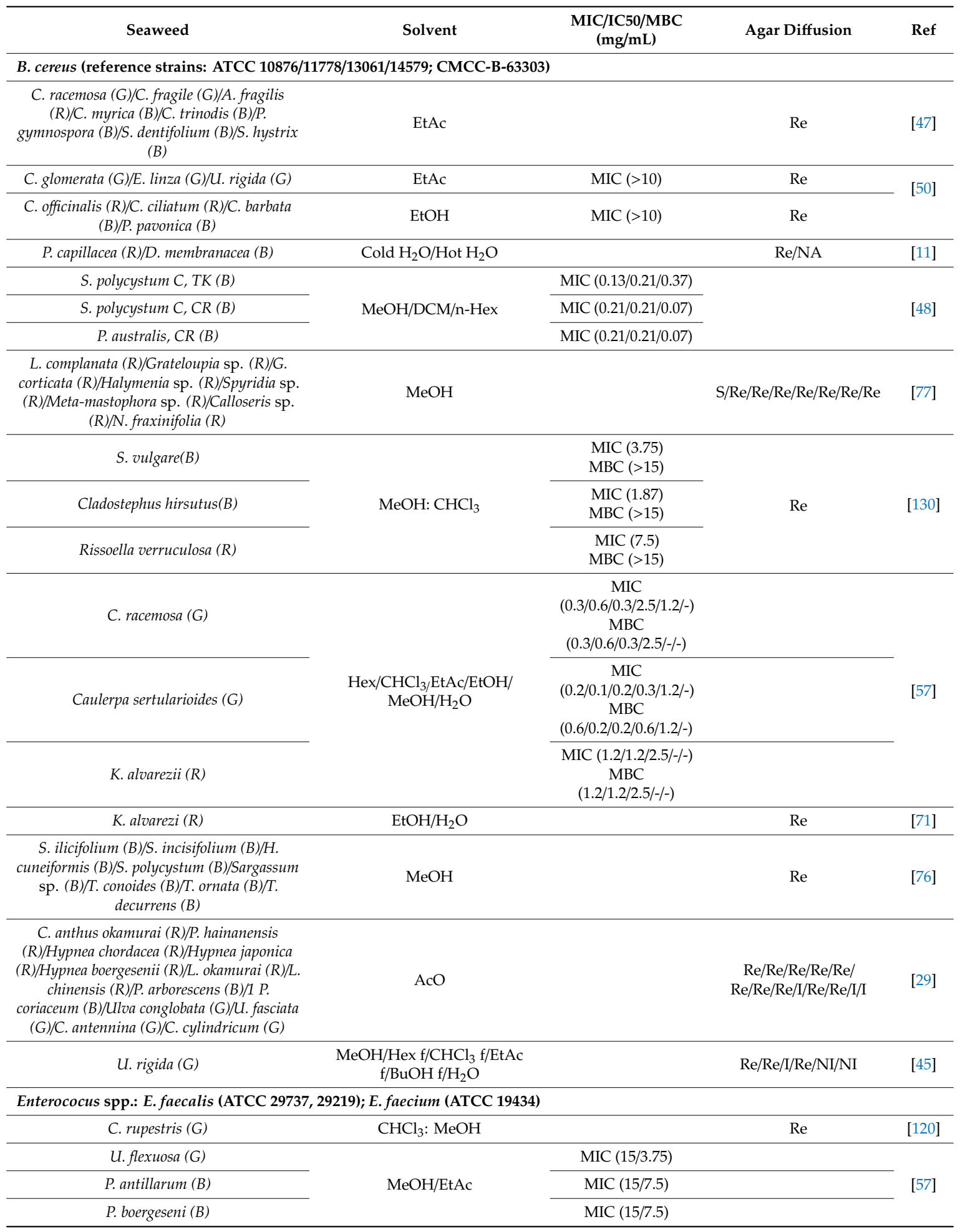


Table 4. Cont

\begin{tabular}{|c|c|c|c|c|}
\hline Seaweed & Solvent & $\begin{array}{l}\mathrm{MIC} / \mathrm{IC} 50 / \mathrm{MBC} \\
(\mathrm{mg} / \mathrm{mL})\end{array}$ & Agar Diffusion & Ref \\
\hline \multirow{2}{*}{ U. rigida $(G)$} & $\mathrm{DCM}$ & & $\mathrm{Re} / \mathrm{I}$ & \multirow{2}{*}{ [80] } \\
\hline & DCM:MeOH & & $\mathrm{Re} / \mathrm{I}$ & \\
\hline $\begin{array}{l}\text { C. nodosa }(G) / \text { H. tuna }(G) / C \text {. bursa }(G) / C \text {. } \\
\text { barbata }(B)\end{array}$ & DCM:MeOH & & $\mathrm{N} / \mathrm{D}$ & [51] \\
\hline $\begin{array}{l}\text { H. musciformis }(G) / S \text {. lomentaria }(B) / S \text {. } \\
\text { filamentosa }(R) / P \text {. pavonica }(B) / C . \\
\text { mediterranea }(B)\end{array}$ & $\mathrm{MeOH}$ & & $\mathrm{ND} / \mathrm{Re} / \mathrm{ND} / \mathrm{Re} / \mathrm{ND} / \mathrm{ND}$ & [54] \\
\hline U. rigida $(G)$ & $\begin{array}{c}\mathrm{Hex} f / \mathrm{CHCl}_{3} \mathrm{f} / \mathrm{EtAc} \mathrm{f} / \mathrm{BuOH} \\
\mathrm{f} / \mathrm{H}_{2} \mathrm{O} \mathrm{f}\end{array}$ & & $\mathrm{ND} / \mathrm{I} / \mathrm{Re} / \mathrm{ND} / \mathrm{ND}$ & [45] \\
\hline Grateloupia doryphore $(R)$ & $\mathrm{MeOH} / \mathrm{EtAc} / \mathrm{EtOH}$ & & $\operatorname{Re}$ & [68] \\
\hline D. membranacea (B) & $\begin{array}{c}\mathrm{MeOH}: \mathrm{DCM} / \mathrm{EtOH} \text { f/AcO } \\
\text { f/MeOH/DCM } \\
\text { f/MeOH:DCM/EtOH f/AcO } \\
\text { f/MeOH:DCM f }\end{array}$ & & $\mathrm{I} / \mathrm{S} / \mathrm{S} / \mathrm{Re} / \operatorname{Re} / \operatorname{Re} / \operatorname{Re} / \operatorname{Re}$ & [28] \\
\hline \multicolumn{5}{|c|}{ L. monocytogenes (reference strains: NCTC 11994; ATCC 19115) } \\
\hline $\begin{array}{c}\text { C. glomerata }(G) / \text { E. linza }(G) / \text { U. rigida }(G) / C \text {. } \\
\text { officinalis }(R) / C \text {. ciliatum }(R) / C \text {. barbata }(B) / P \text {. } \\
\text { pavonica }(B)\end{array}$ & $\mathrm{EtOH}$ & $\begin{array}{c}\mathrm{MBC} \\
(>1.25 />2.2 />10 / \\
>2.5 />2.5 />2.5 />1.25)\end{array}$ & $\operatorname{Re}$ & [50] \\
\hline U. rigida $(G)$ & $\mathrm{Hex} / \mathrm{CHCl}_{3} \mathrm{f}$ & & $\mathrm{S} / \operatorname{Re}$ & [45] \\
\hline D. membranacea (B) & $\mathrm{H}_{2} \mathrm{O}$ & & I & [59] \\
\hline \multirow{6}{*}{ H. elongata (B) } & $\mathrm{H}_{2} \mathrm{O}$ & & $\operatorname{Re}$ & \multirow{6}{*}{ [30] } \\
\hline & $20 \% \mathrm{MeOH}$ & & $\operatorname{Re}$ & \\
\hline & $40 \% \mathrm{MeOH}$ & & $\operatorname{Re}$ & \\
\hline & $60 \% \mathrm{MeOH}$ & & $\operatorname{Re}$ & \\
\hline & $80 \% \mathrm{MeOH}$ & & $\operatorname{Re}$ & \\
\hline & $\mathrm{MeOH}$ & & $\operatorname{Re}$ & \\
\hline $\begin{array}{l}\text { C. glomerata }(G) / U \text {. rigida }(G) / E \text {. linza }(G) / C \text {. } \\
\text { barbata }(B) / P \text {. pavonica }(B) / C \text {. ciliatum }(R) / C \text {. } \\
\text { officinalis }(R)\end{array}$ & $\mathrm{EtOH}$ & & $\mathrm{I} / \mathrm{Re} / \operatorname{Re} / \operatorname{Re} / \operatorname{Re} / \operatorname{Re} / \operatorname{Re}$ & [50] \\
\hline \multicolumn{5}{|c|}{ Streptococcus spp. (S. agalactiae; S. pneumoniae; S. suis; S. aureus; S. mutans; S. pyogenes) } \\
\hline L. brandenii $(R)$ & PeEt:DCM $(6: 4) \mathrm{f}$ & & $\operatorname{Re}$ & [154] \\
\hline C. rupestris $(G)$ & DCM:MeOH & & $\operatorname{Re}$ & [120] \\
\hline L. papillosa $(R)$ & DCM/(DCM:MeOH)/MeOH/ $\mathrm{H}_{2} \mathrm{O}$ & & $\operatorname{Re} / \operatorname{Re} / \operatorname{Re}$ & [155] \\
\hline $\begin{array}{l}\text { H. cuneiformis, (B)/T. ornata (B)/T. conoides } \\
\text { (B)/S. polycystum. (B)/S. ilicifolium (B)/S. } \\
\text { incisifolium (B)/T. decurrens }(B) / \text { Sargassum } \\
\text { sp. (B) }\end{array}$ & $\mathrm{MeOH}$ & & $\operatorname{Re}$ & [76] \\
\hline L. complanata $(R)$ & $\begin{array}{l}\text { MeOH/DCM f/(Pentene:DIEt) } \\
\text { f/MeOH f }\end{array}$ & & $\mathrm{S} / \operatorname{Re} / \operatorname{Re} / \operatorname{Re}$ & [77] \\
\hline $\begin{array}{c}\text { G. corticata }(R) / \text { Halymenia sp. }(R) / \text { Spyridia } \\
\text { sp. }(R) / \text { Meta-mastophora sp. }(R) / \text { Calloseris } \\
\text { sp. }(R) / \text { N. fraxinifolia }(R)\end{array}$ & $\mathrm{MeOH}$ & & $\operatorname{Re}$ & [77] \\
\hline Ulva armoricana $(G)$ & SPoly f & MIC (6.25) & & [156] \\
\hline E. $\operatorname{linza}(G)$ & $\mathrm{EtOH} / \mathrm{MeOH} / \mathrm{AcO}$ & & $\mathrm{Re} / \mathrm{Re} / \mathrm{I}$ & [157] \\
\hline $\begin{array}{l}\text { T. conoides }(B) / P \text {. gymnospora }(B) / S \text {. } \\
\text { tenerrimum }(B)\end{array}$ & $\mathrm{MeOH} / \mathrm{AcO} / \mathrm{PeEt} / \mathrm{EtOH} / \mathrm{EtAc} / \mathrm{CHC}$ & $1_{3 /} \mathrm{DiEt}$ & $\operatorname{Re}$ & [101] \\
\hline G. rugosa $(R)$ & \multirow{2}{*}{ 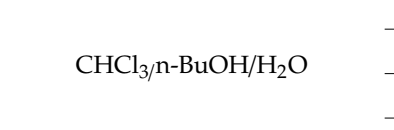 } & $x^{2}$ & $\mathrm{Re} / \mathrm{I} / \mathrm{S}$ & \multirow{2}{*}{ [97] } \\
\hline L. hawaiiana $(R)$ & & 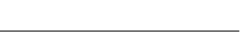 & S/NI/NI & \\
\hline
\end{tabular}

Seaweed information: (G)—green algae (Chlorophytes); (R)—red algae (Rhodophytes); (B)—brown algae (Phaeophyta); nd-not determined. Solvents: AcO-acetone; EtOH-ethanol; $\mathrm{MeOH}-$ methanol; DCM-dichloromethane; EtAc-ethylacetate; $\mathrm{H}_{2} \mathrm{O}$-water; Hex-hexane; $\mathrm{CO}_{2}$-carbon dioxide; DMSO-dimethilsulfoxide; $\mathrm{CHCl}_{3}$ —chloroform; $\mathrm{BuOH}$-butanol; PeEt-petroleum ether; DIEt—diethyl Ether; PolySA—polysaccharides agar; f-fraction. Agar diffusion: Re-resistant $\leq 14 \mathrm{~mm}$; I-intermediate 15-19 $\mathrm{mm}$; S-susceptible $\geq 20 \mathrm{~mm}$ [32]; vs.-very strong; NI-no inhibition. 


\section{Incorporation to the Food Industry}

Nowadays, many molecules have been recognized as permitted additives. They mostly avoid food spoilage induced by oxidant reactions, microbial growth and/or browning processes. Some of the permitted additives with antioxidant capacity are ascorbates, tocopherols, gallates, butylates, lactates, citrates or phosphates, among many others [158]. Those additives considered to exert microbial inhibition growth include acetic, malic, lactic, benzoic, sorbic and propionic acids and some of their salts, as well as parabens. Sulfites are the most used additives for avoiding food browning caused by any chemical or enzymatic reactions. Nevertheless, several natural compounds represent a current alternative to the use of chemical anti-browning ingredients, such as erythorbic acid, cysteine, 4-hexylresorcinol and some phenolic acids [159]. In fact, this is the current trend in the food industry-replacement of chemically synthesized compounds with natural ones. Consumers' claims have prompted this shift due to the side effects related to the consumption of chemically synthesized molecules. Currently, macroalgae represent a promising source of natural molecules with a variety of recognized bioactivities such as antioxidant and anti-microbial, among many others. The high content in polyphenols, such as the phlorotannins that can reach up to $15 \%$ of the dry matter, or pigments, such as carotenoids and chlorophylls, are mainly responsible for their antioxidant activities [160]. It is also known that the diversity of polysaccharides present in macroalgae, carrageenans and agar from red algae, fucoidans from brown and ulvans from green ones, possess antibacterial capacities [161]. Apart from their richness in biomolecules and bioactivities, macroalgae have been demonstrated to represent a cheap, available and eco-friendly source of compounds, which results in being very interesting for the food industry $[160,162,163]$. Currently, algae extracts have been evaluated as food additives for food preservation, as ingredients for creating biodegradable films or core ingredients in active packaging with several functions such as anti-biofilm or anti-fouling agents, as shown in Figure 1.

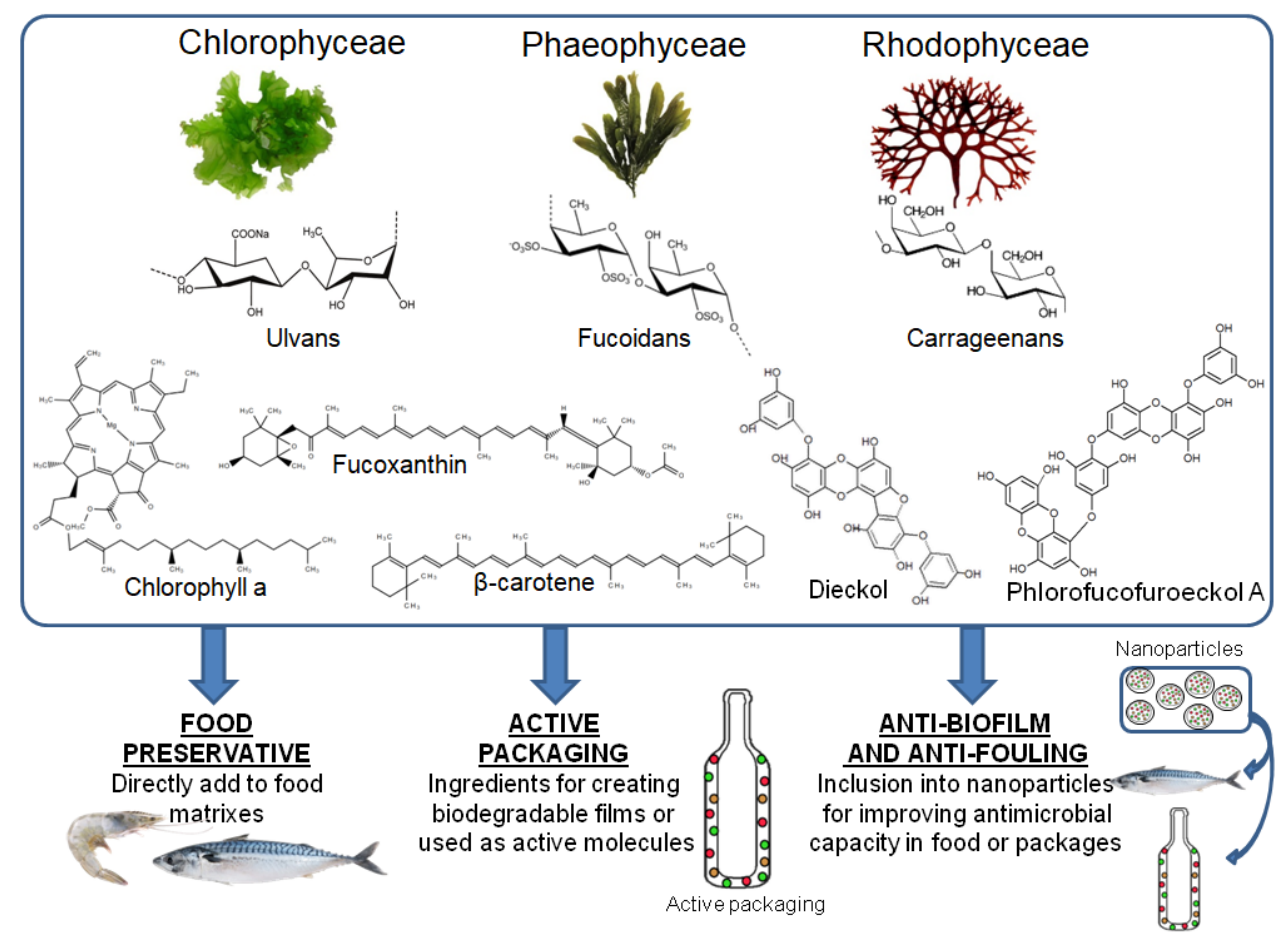

Figure 1. Food applications of macroalgae extracts. Green (Chlorophyceae), brown (Phaeophyceae) and red (Rhodophyceae) macroalgae biomolecules can be used for: (a) their direct application into food products, (b) the development of biodegradable packages and/or for their incorporation as active ingredients into films (active packaging), (c) for their inclusion into encapsulation systems that can be further used for their application in food matrixes or active packaging. 


\subsection{Food Preservative}

The use of algal extracts by the food industry for improving the conservation of different food matrixes has been used for more than two centuries [164]. For instance, polyphenols and polysaccharides from Porphyra yezoensis extended the shelf-life of Litopenaeus vannamei when refrigerated. The efficiency of conservation was evaluated through several parameters considered for the determination of food spoilage: colony-forming units (CFU), $\mathrm{pH}$, total volatile basic nitrogen (TVB-N) or thiobarbituric acid (TBA), among others. Results demonstrated that the application of these algal compounds were able to inhibit the shrimp spoilage and prolong its shelf-life for a further 3-4 days [164]. Similarly, extracts from G. verrucosa also permitted to extend the sensory score of Indian mackerel (Rastrelliger kanagurta) by 4 days when compared to the traditional ice bath storage. As in the previous research, different spoilage parameters such as TVB-N, $\mathrm{pH}$, bioamines concentration and microbial proliferation were studied. The presence of the polyphenols from the alga significantly inhibited the microbial growth, delayed the increment of spoilage markers and prevented the formation of biogenic amines [165]. Very similar results were observed when using C. compressa extracts for conserving chilled Trachurus trachurus. The presence of the alga inhibited the microbial activity and the chemical spoilage markers while reducing those related to lipid hydrolysis and oxidation [166]. Other work also stated the preservative ability of polyphenols obtained from Fucus spiralis and $U$. lactuca when incorporated as a canning medium for Scomber colias. The presence of the algae inhibited the breakdown of free fatty acids and the increment in the peroxide value, especially for samples treated with F. spiralis [167]. Therefore, the incorporation of algae compounds into fish or shellfish matrixes provides extended storage periods while reducing the microorganism growth rate, which ultimately improves food safety.

\subsection{Active Packaging}

The application of active packaging is an innovative solution for improving the storage time of food products. This approach consists of activating a film material by the inclusion of molecules with recognized bioactivities. Usually, the two main issues concerning any food matrix are the oxidation reactions and the microbial spoilage, thus the main bioactivities expected to accomplish by active packaging are antioxidant and antimicrobial properties. Macroalgae have served as a source of natural biomolecules with antioxidant and antimicrobial capacities to create active packaging systems but also as a source of biomaterials for creating biodegradable films.

The extraction of algae molecules has been widely optimized for their further incorporation into films. For instance, differential heat treatments of Mastocarpus stellatus with/without enzymatic hydrolysis permitted to obtain several extracts with strong antioxidant activity due to the presence of polyphenols or higher contents of $k / \mathrm{l}$-hybrid carrageenan or protein. The mixture of three of these extracts, using glycerol as plasticizer, provided a film characterized by the presence of proteins and sulfated compounds that presented high water resistance and appropriate mechanical properties. Additionally, this film possessed effective antioxidant activity, quantified as $70.60 \pm 0.47 \mathrm{mg}$ of vitamin C equivalents per g (ABTS), $1.16 \pm 0.04 \mu \mathrm{mol}$ of $\mathrm{Fe}^{2+}$ per g (FRAP), $41.32 \pm 3.19 \mathrm{mg}$ gallic acid equivalent per $\mathrm{g}$ of sample (Folin-reactive substances) [168,169].

A polylactic acid-based biodegradable film was incorporated with $8 \%$ of $F$. spiralis extract and $1 \%$ of sorbic acid. Its protective effect was evaluated during the refrigerated storage of megrim (Lepidorhombus whiffiagonis). For quantifying the preservative efficiency of the film, the following measurements were performed: trimethylamine-N and CFU account for the antimicrobial capacity, and peroxide values and fluorescent compound formation for the antioxidant activity. These quantifications permitted the determination that the film successfully extended the shelf-life of the product up to 11 days, maintaining the organoleptic properties [170]. The presence of $8 \%$ of algal extracts and $1 \%$ of sorbic acid in PLA has been further supported. A work used up to five different genera, Fucus, Bifurcaria, Gracilaria, Ulva and Ascophyllum, to test their antimicrobial capacity against B. cereus, B. subtilis, S. aureus, K. pneumoniae, Pseudomonas fluorescens, E. coli, Aeromonas hydrophyla, Vibrio alginolyticus and 
V. parahaemolyticus. The most effective algae were Fucus followed by Bifurcaria. Additionally, sorbic acid has been demonstrated to be effective against yeast and molds [171].

Among the molecules present in macroalgae, alginates and fucoidans mostly extracted from brown macroalgae, such as Sargassum natans, S. latifolium or L. japonica, have been repeatedly used for the developing of biodegradable films [172-174]. Even though brown algae are the main target for obtaining ingredients for creating films, red algae such as Gracilaria lemaneiformis has also demonstrated to possess a protein and polysaccharide composition with promising filming properties [175]. These bio packages have been differently designed using just algae-derived polysaccharides for the development of the film or algae extracts combined with other vegetal molecules such as chitosan [172-176]. The antimicrobial activity of algae polysaccharides can be reinforced with the presence of chitosan since it has been recognized to possess antimicrobial capacity [177]. Furthermore, the phenolic composition of algae extracts contributes to the antioxidant effect. Therefore, films created using algae molecules can be considered themselves as active packaging since they offer a physical barrier while providing biological activities.

Therefore, the use of algae compounds allows the creation of a huge variety of active packaging, which represents an innovative tool for conserving food products. Algae-based active packaging can provide biodegradable films that are free of synthetic molecules showing a positive environmental and economic impact. They would reduce the utilization of single-use plastics and would represent an eco-friendly and safe package that may improve consumers' acceptance.

\subsection{Anti-Biofilm and Anti-Fouling}

Bacteria have the ability to get attached to solid supports where they form structured communities also known as biofilms [178]. Food can represent a perfect growth media for microorganisms and thus, an easy target for the development of biofilms. Due to the negative economic and health impact that the development of biofilms has for the food industry, new packaging alternatives have been designed. The use of antibiotics has been strongly associated with pathogenic bacteria resistance, which means a global health threat [179]. As aforementioned, macroalgae polysaccharides have been described to possess antibacterial activity $[161,178]$. However, other compounds present in different algae species have shown antifouling and antibacterial abilities. Polyphenols present in brown algae such as Fucus, Bifurcaria, Cystoseira or Sargassum possess antibacterial activity such as fatty acids from red algae genera Laurencia and Chondrus. Pigments, such as chlorophyll or $\beta$-carotene, contained in green algae such as Ulva have been described as antibacterial and antifouling [180]. Algae extracts have been considered for their application as antifouling or anti-biofilm coating compounds. As explained above, the incorporation of polysaccharides, such as those extracted from Fucus and Bifurcaria, into films, such as PLA, provides antimicrobial capacity, which can ultimately avoid biofilm creation $[170,171]$. Additionally, Gelidium corneum active principles were encapsulated into silver nanoparticles, which showed high antimicrobial activity at very low MICs for Candida albicans $(0.5 \mu \mathrm{g} / \mathrm{mL})$ and for E. coli $(0.3 \mu \mathrm{g} / \mathrm{mL})$. In fact, the antibiofilm efficacy of the $2 \mu \mathrm{g} / \mathrm{mL}$ nanoparticles was demonstrated to be higher than a $70 \%$ threat [179].

The potential of macroalgae compounds to prevent biofilm apparition in food matrixes is still under development. Nevertheless, the application of algae extracts in different films, both free and encapsulated, represents a very promising tool for avoiding anti-fouling and bacterial spoilage without requiring the use of harmful antibiotics.

\section{Food Safety and Quality Control Enhancement Using Antimicrobials from Seaweeds}

The main concern for authorities and professionals in the food supply chain is to guarantee food quality and safety [181]. The keeping of the organoleptic characteristics and the nutritional and chemical composition of foods is influenced by the elimination of foodborne microorganisms. Microbial risk is the most important factor in food safety. Microorganisms may affect food safety both through direct ingestion of pathogens present in food and by the ingestion of the microbial 
toxins excreted in foods by microorganisms during handling before processing [182].Food systems present high susceptibility to microbial contamination, which may diminish their quality properties and therefore, their nutritional value [183].

Hence, the main problem regarding food safety is foodborne pathogens [184]. Food safety and quality control can be enhanced using antimicrobial agents that can be present naturally or incorporated ad hoc in foods to inhibit microbial growth or cause the death of microorganisms [185]. Foodborne microorganisms are responsible for diseases with a high impact on public health and currently, they are an important concern for the health authorities. In addition to causing infections and intoxications through contaminated foods, pathogenic bacteria are responsible for the food deterioration during storage and distribution with a high impact on food quality, shelf-life and food loss. Therefore, the search for solutions to avoid the deterioration of food caused by pathogenic and spoilage microorganisms has focused the efforts of the food industry, in order to improve food safety and quality [185]. Figure 2 shows an illustration where the general concepts related to food safety and quality control are summarized in a visual form.

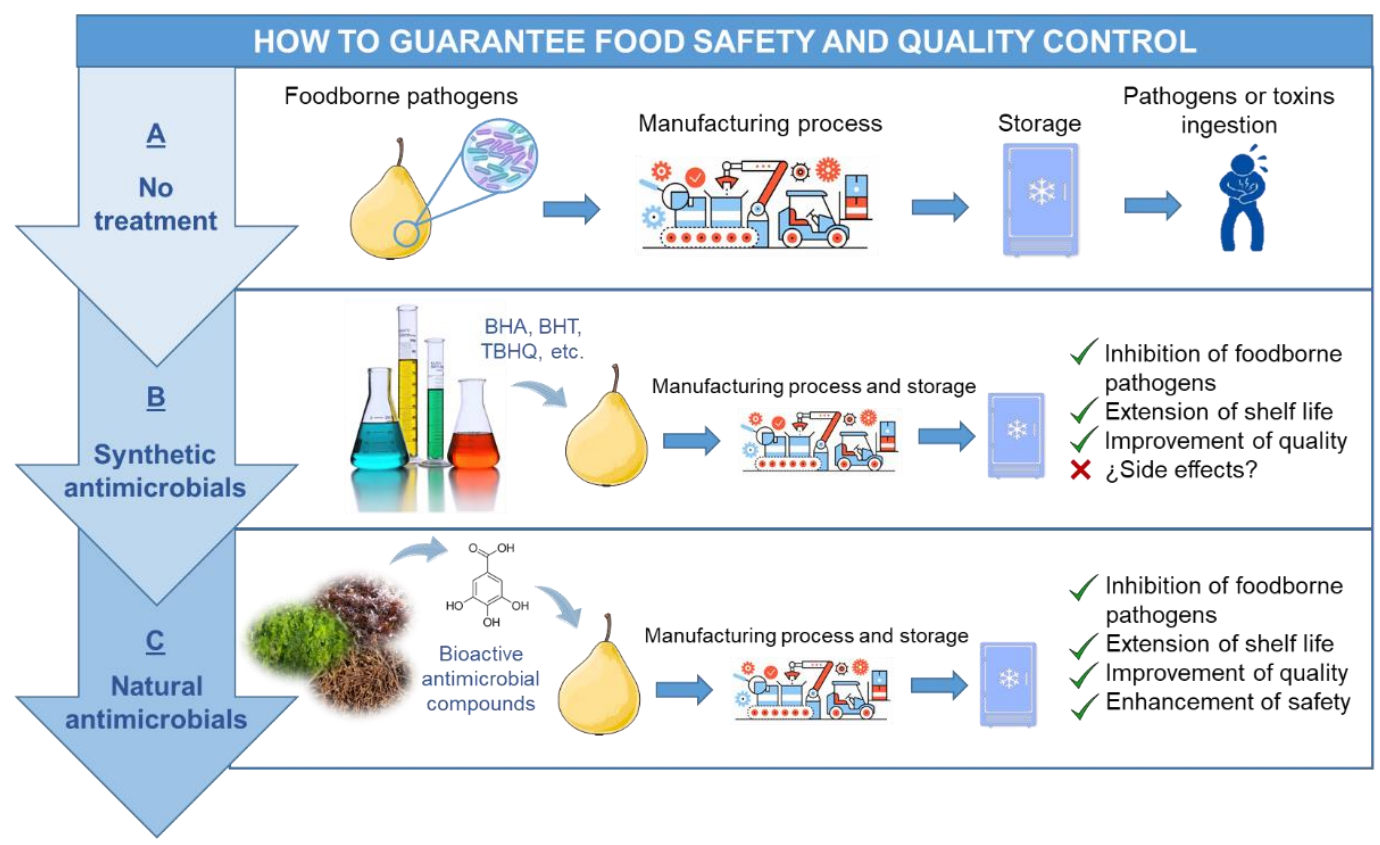

Figure 2. Enhancement of food safety and quality control of fresh and ready-to-eat foods using natural antimicrobials from seaweeds.

Two facts have encouraged the search of antimicrobials in the food industry, on one hand, conventional methods to preserve foods present less efficiency to reduce or inhibit the proliferation of foodborne pathogens and on the other hand, the increased demand for minimally processed foods. The application of antimicrobials is a new strategy to increase the shelf life of food products and overcome the problems of food quality and safety [184]. Preservation of food safety and quality has been extensively carried out by the food industry using synthetic antimicrobials (sodium benzoate, sodium nitrite and sorbic acid) and synthetic antioxidants (butylated hydroxyanisole (BHA), butylated hydroxytoluene (BHT) and tert-butyl hydroxyquinone (TBHQ)). However, in the last few years, it has been reported that these synthetic compounds could have toxic and carcinogenic effects for the consumer $[184,186]$. Additionally, the interest in antimicrobials from natural sources has also increased due to the incorrect use of antibiotics, which has provoked the development of multidrug-resistant microorganisms, including foodborne pathogens that exhibit resistance to the current antibiotics and are not further affected by the conventional food processing and preservation techniques. From a point of view of food safety and quality control, such natural antimicrobials are the key to solve 
the drawbacks described above [187]. Nowadays, the increase in consumer demand for minimally processed foods with natural additives and consumer concern about food safety and quality has encouraged the search for alternative natural bio preservatives with antimicrobial properties $[184,186]$.

In this context, in recent years, the use of preservatives from natural sources to avoid or decrease the growth of foodborne microorganisms is a promising approach to replace synthetic ones [184]. Antimicrobial extracts from seaweeds are outlined as alternatives to the synthetic preservatives in the food industry to keep or improve food safety and quality [188]. The possibility of incorporating such extracts in perishable food products, such as refrigerated ready-to-eat foods, could expand their shelf-life, diminish or remove pathogenic bacteria and at the same time, enhance the perception of the consumer to processed food by substituting synthetic with natural antimicrobial agents [189]. Previous sections collect several works about the antimicrobial properties of different seaweeds, which would have potential applications in improving the safety and quality of foods.

\section{Future Perspectives and Conclusions}

Before the creation of modern antibiotics, humanity relied on plants and plant extracts to treat infections and other diseases. Modern antibiotics brought a new age of medicine. Currently, society is facing a major threat to the health of the populations as some bacteria have developed resistance to contemporary antibiotics. This demands a different approach to the problem, perhaps based on materials that were traditionally used but applying modern techniques. Throughout this article, we reviewed the antibacterial effect that some algae extracts can have on several foodborne pathogens responsible for worldwide diseases. There is a clear trend in the current market for the consumption of more eco-friendly and natural products and their incorporation not only in consumers' diets but also in their personal care and overall health showing a potential market for algae-based medicine [189]. Another concern is that invasive algae are threatening ecosystems. These algae can have an antibacterial effect and be potentially used in treatments, thus reducing the amount of this invasive species and helping to reduce their environmental impact.

Algae and alga extracts are also described as able to improve food quality when they are incorporated in feedstock [190], helping to control infections in fish farms [190]. Nowadays, food safety, functional foods and non-traditional diets are very important issues and algae incorporation in food products is already a reality [191,192]. Therefore, macroalgae are an easily available resource that has great potential as the origin of new active biomolecules capable of positively contributing to the control of foodborne bacteria [193]. This contribution could be used as newfound food preservatives, active packaging or anti-fouling and anti-biofilm agents as discussed previously. Further efforts should be made in the pursuit of appealing organoleptic characteristics as algae and algae compounds could have a very interesting role in food safety in the nearby future.

The present review focused on the possible utilization of algae-based bioactive compounds (such as alkaloids, proteins, polysaccharides, polyphenols, tannins, catechin, fatty acids) $[20,72,88]$ against the proliferation of foodborne pathogens.

There is a considerable amount of data on different algae species and their ability to minimize and even stop bacterial growth. However, reported works show that there are several issues strongly affecting algae extract performance. Extraction conditions and the solvent selection seem to play an important role in the performance of the algae extracts as antimicrobial agents. Nevertheless, even though the pursuit for clean and safe extraction techniques continues, some toxic chemicals such as chloroform, are still used as solvent possibilities. It is also noteworthy that environmental conditions and seaweed harvest location also play a part in the process, being responsible for the variations in the antimicrobial effectiveness of the algae extracts. The demand for natural foods is a worldwide trend, and in that sense, algae are an important resource due to their nutritional properties and particularly due to the bioactive molecules present in their composition. As a result, antimicrobial compounds extracted from algae have shown potential, fundamentally as food preservatives of direct application but also as part of the formulation of active packaging and for other functions such as anti-biofilm 
or anti-fouling. More applications are expected to be developed. Additionally, algal extracts might contribute towards food safety as they have been proven to be active against foodborne pathogens.

Author Contributions: All authors contributed equally. All authors have read and agreed to the published version of the manuscript.

Funding: This research received no external funding.

Acknowledgments: The research was funded by Programa de Cooperación Interreg V-A España-Portugal (POCTEP) 2014-2020 (projects ref. 0181_NANOEATERS_01_E and ref. 0377_IBERPHENOL_6_E) that supports the pre-doctoral grant for C. Jimenez-Lopez; by MICINN supporting the Ramón\&Cajal grant for M. A. Prieto (RYC-2017-22891); by Xunta de Galicia and University of Vigo supporting the post-doctoral grant for M. Fraga-Corral (ED481B-2019/096); by Axudas Conecta Peme (Xunta de Galicia) supporting the IN852A 2018/58 NeuroFood Project; AlgaMar (www.algamar.com) that supports the pre-doctoral grant for C. Lourenço-Lopes; by EcoChestnut Project (Erasmus+ KA202) for supporting the work of M. Carpena; and by the project Bio Based Industries Joint Undertaking (JU) under grant agreement no 888003 UP4HEALTH Project (H2020-BBI-JTI-2019) for supporting the postdoctoral work of P. Gullón. This work also received financial support from REQUIMTE/LAQV, National Funds (FCT, Fundação para a Ciência e Tecnologia) through project UID/QUI/50006/2019 and by FEDER-Fundo Europeu de Desenvolvimento Regional funds through the COMPETE 2020-Operational Programme for Competitiveness and Internationalisation (POCI), and by Portuguese funds through FCT-Fundação para a Ciência e a Tecnologia in the framework of the project POCI-01-0145-FEDER-030240_PTDC/OCE-ETA/30240/2017.

Conflicts of Interest: The authors declare no conflict of interest.

\section{Abbreviations}

$\begin{array}{ll}\text { Generic } & \\ \text { ATCC } & \text { American Type Culture Collection } \\ \text { B } & \text { brown algae (Phaeophyta) } \\ \text { BAA } & \text { Asian Bacterial Bank } \\ \text { CLSI } & \text { Clinical and Laboratory Standards Institute } \\ \text { CFU } & \text { Colony Formation Unix } \\ \text { CMCC } & \text { China Medical Culture Collection Center } \\ \text { DSM } & \text { Deutsche Sammlung von Mikroorganismen } \\ \text { f } & \text { fraction } \\ \text { G } & \text { green algae (Chlorophyta) } \\ \text { FRAP } & \text { Ferric Antioxidant Power } \\ \text { MBC } & \text { minimal bactericide concentration } \\ \text { MIC } & \text { minimal inhibition concentration } \\ \text { MRSA } & \text { methicillin-resistant S. aureus } \\ \text { MTCC } & \text { Microbial Type Culture Collection and Gene Bank } \\ \text { NCIMB } & \text { National Collection of Industrial, Food and Marine Bacteria } \\ \text { nd } & \text { not determined } \\ \text { nt } & \text { not tested } \\ \text { NI } & \text { no Inhibition } \\ \text { PLA } & \text { Polylactic acid } \\ \text { PTCC } & \text { Persian Type Culture Collection } \\ \text { GMP } & \text { good manufacturing practices } \\ \text { HACCP } & \text { Hazard Analysis and Critical Control Point } \\ \text { I } & \text { intermediate 15-19 mm } \\ \text { IC50 } & \text { Half maximal inhibitory concentration } \\ \text { RCM } & \text { The Republic Collection of Microogrganisms } \\ \text { Re } & \text { resistant } \leq 14 \text { mm } \\ \text { SSOP } & \text { sanitation standard operating procedure } \\ \text { R } & \text { red algae (Rhodophyta) } \\ \text { S } & \text { susceptible } \geq 20 \text { mm } \\ & \end{array}$




$\begin{array}{ll}\text { Compounds } & \\ \mathrm{ABTS} & 2,2^{\prime} \text {-azino-bis(3-ethylbenzothiazoline-6-sulfonic acid) } \\ \mathrm{AcO} & \text { acetone } \\ \mathrm{n}-\mathrm{BuOH} & \text { butanol } \\ \mathrm{ClHx} & \text { ciclohexane } \\ \mathrm{CHCl}_{3} & \text { chloroform } \\ \mathrm{CO}_{2} & \text { carbon dioxide } \\ \mathrm{DCM} & \text { dichloromethane } \\ \mathrm{DMSO} & \text { dimethilsulfoxide } \\ \mathrm{DIEt} & \text { diethyl ether } \\ \mathrm{DPPH} & \text { 1,1-diphenyl-2-picryl hydrazyl } \\ \text { EtAc } & \text { ethylacetate } \\ \text { EtOAc } & \text { ethyl ethanoate } \\ \text { EtOH } & \text { ethanol } \\ \mathrm{Hex} & \text { hexane } \\ \mathrm{H}{ }_{2} \mathrm{O} & \text { water } \\ \mathrm{MeOH} & \text { methanol } \\ \text { n-Hex } & \text { n-hexane } \\ \text { PeEt } & \text { petroleum ether } \\ \text { PUFA } & \text { poly-unsaturated fatty acids } \\ \text { PolySA } & \text { polysaccharides agar } \\ \end{array}$

\section{References}

1. Ibrahim, M.; Salman, M.; Kamal, S.; Rehman, S.; Razzaq, A.; Akash, S.H. Algae-Based Biologically Active Compounds. In Algae Based Polymers, Blends, and Composites: Chemistry, Biotechnology and Materials Science; Elsevier Inc.: Amsterdam, The Netherlands, 2017; pp. 155-271, ISBN 9780128123607.

2. Kandale, A.; Meena, A.K.; Rao, M.M.; Panda, P.; Mangal, A.K.; Reddy, G.; Babu, R. Marine Algae: An Introduction, Food Value and Medicinal Uses. J. Pharm. Res. 2011, 4, 219-221.

3. Malhotra, S.; Singh, A.P. Algae, traditional medicine, and pharmacological advances. Int. J. Algae 2008, 10, 299-308. [CrossRef]

4. Aknin, M.; Dogbevi, K.; Samb, A.; Kornprobst, J.M.; Gaydou, E.M.; Miralles, J. Fatty acid and sterol composition of eight brown algae from the Senegalese coast. Comp. Biochem. Physiol. Part B Biochem. 1992, 102B, 841-843. [CrossRef]

5. Frikha, F.; Kammoun, M.; Hammami, N.; Mchirgui, R.; Belbahri, L.; Gargouri, Y.; Miled, N.; Ben, R.F. Chemical composition and some biological activities of marine algae collected in Tunisia. Cienc. Mar. 2011, 37, 113-124. [CrossRef]

6. Michalak, I.; Chojnacka, K. Algae as production systems of bioactive compounds. Eng. Life Sci. 2015, 15, 160-176. [CrossRef]

7. Patra, J.K.; Rath, S.K.; Jena, K.; Rathod, V.K.; Thatoi, H. Evaluation of antioxidant and antimicrobial activity of seaweed (Sargassum sp.) extract: A study on inhibition of glutathione-S-transferase activity. Turk. J. Biol. 2008, 32, 119-125.

8. Agatonovic-kustrin, S.; Ramenskaya, G.; Kustrin, E.; Ortakand, D.B.; Morton, D.W. A new integrated HPTLC-ATR/FTIR approach in marine algae bioprofiling. J. Pharm. Biomed. Anal. 2020, 113488. [CrossRef]

9. Andriani, Z.; Fasya, A.G.; Hanapi, A. Antibacterial Activity of the Red Algae Eucheuma cottonii Extract from Tanjung Coast, Sumenep Madura. Alchemy 2016, 4, 93-100. [CrossRef]

10. Bittkau, K.S.; Neupane, S.; Alban, S. Initial evaluation of six different brown algae species as source for crude bioactive fucoidans. Algal Res. 2020, 45, 101759. [CrossRef]

11. de Borba Gurpilhares, D.; Moreira, T.R.; da Luz Bueno, J.; Cinelli, L.P.; Mazzola, P.G.; Pessoa, A.; Sette, L.D. Algae's sulfated polysaccharides modifications: Potential use of microbial enzymes. Process Biochem. 2016, 51, 989-998. [CrossRef]

12. Patel, S. Therapeutic importance of sulfated polysaccharides from seaweeds: Updating the recent findings. 3 Biotech 2012, 2, 171-185. [CrossRef] 
13. Jimenez-Lopez, C.; Fraga-Corral, M.; Carpena, M.; García-Oliveira, P.; Echave, J.; Pereira, A.G.; Lourenço-Lopes, C.; Prieto, M.A.; Simal-Gandara, J. Agriculture waste valorisation as a source of antioxidant phenolic compounds within a circular and sustainable bioeconomy. Food Funct. 2020. [CrossRef] [PubMed]

14. Fraga-Corral, M.; García-Oliveira, P.; Pereira, A.G.; Lourenço-Lopes, C.; Jimenez-Lopez, C.; Prieto, M.A.; Simal-Gandara, J. Technological application of tannin-based extracts. Molecules 2020, 25, 614. [CrossRef] [PubMed]

15. Li, Y.X.; Wijesekara, I.; Kim, S.K.; Li, Y. Phlorotannins as bioactive agents from brown algae. Process Biochem. 2011, 46, 2219-2224. [CrossRef]

16. Desnoyers, M.; Gilbert, K.; Rousseau, G. Cardioprotective Effects of Omega-3 Polyunsaturated Fatty Acids: Dichotomy between Experimental and Clinical Studies. Mar. Drugs 2018, 16, 234. [CrossRef]

17. Bhagavathy, S.; Sumathi, P.; Jancy Sherene Bell, I. Green algae Chlorococcum humicola- a new source of bioactive compounds with antimicrobial activity. Asian Pac. J. Trop. Biomed. 2011, 1, S1. [CrossRef]

18. Taskin, E.; Ozturk, M.; Taskin, E.; Kurt, O. Antibacterial activities of some marine algae from the Aegean Sea (Turkey). Afr. J. Biotechnol. 2007, 6, 2746-2751.

19. Robinson, I.; Junqua, G.; Van Coillie, R.; Thomas, O. Trends in the detection of pharmaceutical products, and their impact and mitigation in water and wastewater in North America. Anal. Bioanal. Chem. 2007, 387, 1143-1151. [CrossRef]

20. Kini, S.; Divyashree, M.; Mani, M.K.; Mamatha, B.S. Algae and cyanobacteria as a source of novel bioactive compounds for biomedical applications. In Advances in Cyanobacterial Biology; Academic Press: Cambridge, MA, USA, 2020; pp. 173-194.

21. Plaza, M.; Santoyo, S.; Jaime, L.; García-Blairsy Reina, G.; Herrero, M.; Señoráns, F.J.; Ibáñez, E. Screening for bioactive compounds from algae. J. Pharm. Biomed. Anal. 2010, 51, 450-455. [CrossRef]

22. Karthikeyan, S.; Prathima, A.; Periyasamy, M. Characteristics studies on Stoechospermum marginatum, brown marine algae with Al2O3 nanofluid. Mater. Today Proc. 2020, 2-6. [CrossRef]

23. Olaimat, A.N.; Holley, R.A. Factors influencing the microbial safety of fresh produce: A review. Food Microbiol. 2012, 32, 1-19. [CrossRef]

24. Kim, D.H.; Cho, W.I.; Lee, S.J. Fault tree analysis as a quantitative hazard analysis with a novel method for estimating the fault probability of microbial contamination: A model food case study. Food Control 2020, 110, 107019. [CrossRef]

25. Chatterjee, A.; Abraham, J. Microbial Contamination, Prevention, and Early Detection in Food Industry. In Microbial Contamination and Food Degradation; Elsevier: Cambridge, UK, 2018; pp. 21-47.

26. Mani-López, E.; Palou, E.; López-Malo, A. Biopreservatives as Agents to Prevent Food Spoilage. In Microbial Contamination and Food Degradation; Elsevier: Cambridge, UK, 2018; pp. 235-270.

27. Balouiri, M.; Sadiki, M.; Ibnsouda, S.K. Methods for in vitro evaluating antimicrobial activity: A review. J. Pharm. Anal. 2016, 6, 71-79. [CrossRef]

28. Akremi, N.; Cappoen, D.; Anthonissen, R.; Verschaeve, L.; Bouraoui, A. Phytochemical and in vitro antimicrobial and genotoxic activity in the brown algae Dictyopteris membranacea. S. Afr. J. Bot. 2017, 108, 308-314. [CrossRef]

29. Peihang, X.U.; Huaqiang, T.A.N.; Weiguang, J.I.N. Antioxidative and antimicrobial activities of intertidal seaweeds and possible effects of abiotic factors on these bioactivities. J. Oceanol. Limnol. 2018, 36, 2243-2256.

30. Rajauria, G.; Jaiswal, A.K.; Abu-gannam, N.; Gupta, S. Antioxidant and free radical-scavenging capacity of brown seaweed himanthalia elongata from western coast of Ireland. J. Food Biochem. 2012, 37, 1-14. [CrossRef]

31. Sellimi, S.; Maalej, H.; Rekik, D.M.; Benslima, A.; Ksouda, G.; Hamdi, M.; Sahnoun, Z.; Li, S.; Nasri, M.; Hiji, M. Antioxidant, antibacterial and in vivo wound healind properties of laminaran purified from Cystoseira barbata seaweed. J. Biol. Macromol. 2018, 119, 633-644. [CrossRef] [PubMed]

32. CLSI M02-A12. Performance Standards for Antimicrobial Disk; Clinical and Laboratory Standards Institute: Wayne, NJ, USA, 2015; Volume 32, ISBN 1562389858.

33. Wiegand, I.; Hilpert, K.; Hancock, R.E.W. Agar and broth dilution methods to determine the minimal inhibitory concentration (MIC) of antimicrobial substances. Nat. Protoc. 2008, 3, 163-175. [CrossRef]

34. Tenover, F.C. Antibiotic Susceptibility Testing. In Encyclopedia of Microbiology; Elsevier: Amsterdam, The Netherlands, 2009; pp. 67-77. 
35. Croxen, M.A.; Law, R.J.; Scholz, R.; Keeney, K.M.; Wlodarska, M.; Finlay, B.B. Recent Advances in Understanding Enteric Pathogenic Escherichia coli. Clin. Microbiol. Rev. 2013, 26, 822-880. [CrossRef] [PubMed]

36. Ripolles-Avila, C.; Martínez-Garcia, M.; Capellas, M.; Yuste, J.; Fung, D.Y.C.; Rodríguez-Jerez, J. From hazard analysis to risk control using rapid methods in microbiology: A practical approach for the food industry. Compr. Rev. Food Sci. Food Saf. 2020, 19, 1877-1907. [CrossRef]

37. Authority, E.F.S. The European Union One Health 2018 Zoonoses Report. EFSA J. 2019, 17. [CrossRef]

38. Heiman, K.E.; Mody, R.K.; Johnson, S.D.; Griffin, P.M.; Gould, L.H. Escherichia coli O157 Outbreaks in the United States, 2003-2012. Emerg. Infect. Dis. 2015, 21. [CrossRef] [PubMed]

39. Yang, S.-C.; Lin, C.-H.; Aljuffali, I.A.; Fang, J.-Y. Current pathogenic Escherichia coli foodborne outbreak cases and therapy development. Arch. Microbiol. 2017, 199, 811-825. [CrossRef] [PubMed]

40. De Alencar, D.B.; de Carvalho, F.C.T.; Rebouças, R.H.; dos Santos, D.R.; dos Santos Pires-Cavalcante, K.M.; de Lima, R.L.; Baracho, B.M.; Bezerra, R.M.; Viana, F.A.; dos Fernandes Vieira, R.H.S.; et al. Bioactive extracts of red seaweeds Pterocladiella capillacea and Osmundaria obtusiloba (Floridophyceae: Rhodophyta) with antioxidant and bacterial agglutination potential. Asian Pac. J. Trop. Med. 2016, 9, 372-379. [CrossRef]

41. Kosanić, M.; Ranković, B.; Stanojković, T. Brown macroalgae from the Adriatic Sea as a promising source of bioactive nutrients. J. Food Meas. Charact. 2019, 13, 330-338. [CrossRef]

42. Stanojkovic, T.P.; Ranković, B.; Kosanić, M. Evaluation of antioxidant, antimicrobial and anticancer effects of three selected marine macroalgae. Rom. Biotechnol. Lett. 2018, 23, 13804-13813.

43. Otero, P.; Quintana, S.E.; Reglero, G.; Fornari, T.; Garc, R. Pressurized Liquid Extraction (PLE) as an Innovative Green Technology for the Effective Enrichment of Galician Algae Extracts with High Quality Fatty Acids and Antimicrobial and Antioxidant Properties. Mar. Drugs 2018, 16, 156. [CrossRef]

44. Meillisa, A.; Siahaan, E.A.; Park, J.N.; Woo, H.C.; Chun, B.S. Effect of subcritical water hydrolysate in the brown seaweed Saccharina japonica as a potential antibacterial agent on food-borne pathogens. J. Appl. Phycol. 2013, 25, 763-769. [CrossRef]

45. Trigui, M.; Gasmi, L.; Zouari, I. Seasonal variation in phenolic composition, antibacterial and antioxidant activities of Ulva rigida (Chlorophyta) and assessment of antiacetylcholinesterase potential. J. Appl. Phycol. 2013, 319-328. [CrossRef]

46. Kosanić, M.; Ranković, B.; Stanojković, T. Biological potential of marine macroalgae of the genus Cystoseira. Acta Biol. Hung. 2015, 66, 374-384. [CrossRef]

47. Salem, W. Screening for antibacterial activities in some marine algae from the red sea (Hurghada, Egypt). Afr. J. Microbiol. Res. 2011, 5, 2160-2167. [CrossRef]

48. Chong, C.W.; Hii, S.L.; Wong, C.L. Antibacterial activity of Sargassum polycystum C. Agardh and Padina australis Hauck (Phaeophyceae). Afr. J. Biotechnol. 2011, 10, 14125-14131. [CrossRef]

49. Tajbakhsh, S.; Ilkhani, M.; Rustaiyan, A.; Larijani, K.; Sartavi, K.; Tahmasebi, R.; Asayesh, G. Antibacterial effect of the brown alga Cystoseira trinodis. J. Med. Plant Res. 2011, 5, 4654-4657.

50. Ertürk, Ö.; Ta, B. Antibacterial and Antifungal Effects of Some Marine Algae Makale Kodu. Kafkas Univ. Vet. Fak. Derg. 2011, 17, 121-124.

51. Milović, S.; Kundaković, T.; Mačić, V.; Stanković, J.A.; Grozdanić, N.; Đuričić, I.; Stanković, I.; Stanojković, T. Anti $\alpha$-glucosidase, antitumour, antioxidative, antimicrobial activity, nutritive and health protective potential of some seaweeds from the Adriatic coast of Montenegro. Farmacia 2017, 65, 731-740.

52. Osman, N.A.H.K.; Siam, A.A.; El-manawy, I.M.; Jeon, Y. Anti-microbial and Anti-diabetic Activity of Six Seaweeds Collected from the Red Sea, Egypt. Catrina Int. J. Environ. Sci. 2019, 19, 55-60.

53. Alves, C.; Pinteus, S.; Simões, T.; Horta, A.; Silva, J.; Tecelão, C.; Pedrosa, R. Bifurcaria bifurcata: A key macro-alga as a source of bioactive compounds and functional ingredients. Int. J. Food Sci. Technol. 2016, 51, 1638-1646. [CrossRef]

54. Taskin, E.; Caki, Z.; Ozturk, M.; Taskin, E. Assessment of in vitro antitumoral and antimicrobial activities of marine algae harvested from the eastern Mediterranean sea. Afr. J. Biotechnol. 2010, 9, 4272-4277. [CrossRef]

55. Zeid, A.H.A.; Aboutabl, E.A.; Sleem, A.A.; El-rafie, H.M. Water soluble polysaccharides extracted from Pterocladia capillacea and Dictyopteris membranacea and their biological activities. Carbohydr. Polym. 2014, 113, 62-66. [CrossRef] 
56. Al Khazan, M.M.; Omar, H.H.; Gumgumjee, N.M.; Shiekh, H.M.; El-Gendy, A.M. Marine macroalgae as a potential source of bioactive natural products with antibacterial activity. Main Gr. Chem. 2016, 15, 139-151. [CrossRef]

57. Ambreen, A.; Hira, K.; Ruqqia, A.; Sultana, V. Evaluation of biochemical component and antimicrobial activity of some seaweeeds occurring at Karachi coast. Pak. J. Bot. 2012, 44, 1799-1803.

58. Omar, H.H.; Shiekh, H.M.; Gumgumjee, N.M. Antibacterial activity of extracts of marine algae from the Red Sea of Jeddah, Saudi Arabia. Afr. J. Biotechnol. 2012, 11, 13576-13585. [CrossRef]

59. Aoun, Z.B.; Said, R.B.; Farhat, F. Anti-inflammatory, antioxidant and antimicrobial activities of aqueous and organic extracts from Dictyopteris membranacea. Bot. Mar. 2010, 53, 259-264. [CrossRef]

60. Karima, S.; Fatiha, B. Study of the antimicrobial activity of four algerian marin algae species. In Microbes in Applied Research; World Scientific: Singapore, 2012; pp. 578-581.

61. Arulkumar, A.; Rosemary, T.; Paramasivam, S.; Rajendran, R.B. Phytochemical composition, in vitro antioxidant, antibacterial potential and GC-MS analysis of red seaweeds (Gracilaria corticata and Gracilaria edulis) from Palk Bay, India. Biocatal. Agric. Biotechnol. 2018, 15, 63-71. [CrossRef]

62. Omar, H.H.; Al-Judaibiand, A.; El-Gendy, A. Antimicrobial, antioxidant, anticancer activity and phytochemical analysis of the red alga, laurencia papillosa. Int. J. Pharmacol. 2018, 14, 572-583. [CrossRef]

63. Manilal, A.; Sujith, S.; Sabarathnam, B.; Kiran, G.S.; Selvin, J.; Shakir, C.; Lipton, A.P. Biological activity of the red alga Laurencia brandenii. Acta Bot. Croat. 2011, 70, 81-90. [CrossRef]

64. Dos Santos Amorim, R.D.N.; Rodrigues, J.A.G.; Holanda, M.L.; Quinderé, A.L.G.; de Paula, R.C.M.; Melo, V.M.M.; Benevides, N.M.B. Antimicrobial effect of a crude sulfated polysaccharide from the red seaweed gracilaria ornata. Braz. Arch. Biol. Technol. 2012, 55, 171-181. [CrossRef]

65. Assaw, S.; Rosli, N.L.; Azmi, N.A.M.; Mazlan, N.W.; Ismail, N. Antioxidant and antibacterial activities of polysaccharides and methanolic crude extracts of local edible red seaweed Gracilaria sp. Malays. Appl. Biol. 2018, 47, 135-144.

66. Mashjoor, S.; Yousefzadi, M.; Esmaeili, M.A.; Rafiee, R. Cytotoxicity and antimicrobial activity of marine macro algae (Dictyotaceae and Ulvaceae) from the Persian Gulf. Cytotechnology 2016, 68, 1717-1726. [CrossRef]

67. Sirbu, R.; Stanciu, G.; Tomescu, A.; Ionescu, A.M.; Cadar, E. Evaluation of antioxidant and antimicrobial activity in relation to total phenolic content of green algae from Black Sea. Rev. Chim. 2019, 70, 1197-1203. [CrossRef]

68. Abdel-Latif, H.H.; Shams El-Din, N.G.; Ibrahim, H.A.H. Antimicrobial activity of the newly recorded red alga Grateloupia doryphora collected from the Eastern Harbor, Alexandria, Egypt. J. Appl. Microbiol. 2018, 125, 1321-1332. [CrossRef] [PubMed]

69. Goecke, F.; Labes, A.; Wiese, J.; Imhoff, J.F. Dual effect of macroalgal extracts on growth of bacteria in Western Baltic Sea. Rev. Biol. Mar. Oceanogr. 2012, 47, 75-86. [CrossRef]

70. Abdel-Raouf, N.; Mohamed, H.; Mostafa, S.; Ibraheem, I. Controlling of Microbial Growth by Using Cystoseira barbata Extract. Egypt. J. Bot. 2017, 57, 469-477. [CrossRef]

71. Bhuyar, P.; Rahim, M.H.; Sundararaju, S.; Maniam, G.P.; Govindan, N. Antioxidant and antibacterial activity of red seaweed; Kappaphycus alvarezii against pathogenic bacteria. Glob. J. Environ. Sci. Manag. 2020, 6, 47-58. [CrossRef]

72. Munir, N.; Rafique, M.; Altaf, I.; Sharif, N.; Naz, S. Antioxidant and antimicrobial activities of extracts from selected algal species. Bangladesh J. Bot. 2018, 47, 53-61.

73. Jassbi, A.R.; Mohabati, M.; Eslami, S.; Sohrabipour, J.; Miri, R. Biological activity and chemical constituents of red and brown algae from the Persian Gulf. Iran. J. Pharm. Res. 2013, 12, 339-348. [CrossRef] [PubMed]

74. Shanmugam, S.K.; Kumar, Y.; Yar, K.M.S.; Gupta, V.; de Clercq, E. Antimicrobial and cytotoxic activities of Turbinaria conoides (J.Agardh) Kuetz. Iran. J. Pharm. Res. 2010, 9, 411-416. [CrossRef]

75. Selim, S.; Amin, A.; Hassan, S.; Hagazey, M. Antibacterial, cytotoxicity and anticoagulant activities from Hypnea esperi and Caulerpa prolifera marine algae. Pak. J. Pharm. Sci. 2015, 28, 525-530.

76. Rahelivao, M.P.; Gruner, M.; Andriamanantoanina, H.; Bauer, I.; Knölker, H.-J. Brown Algae (Phaeophyceae) from the Coast of Madagascar: Preliminary Bioactivity Studies and Isolation of Natural Products. Nat. Prod. Bioprospect. 2015, 5, 223-235. [CrossRef]

77. Rahelivao, M.P.; Gruner, M.; Andriamanantoanina, H.; Andriamihaja, B.; Bauer, I.; Knölker, H. Red Algae (Rhodophyta) from the Coast of Madagascar: Preliminary Bioactivity Studies and Isolation of Natural Products. Mar. Drugs 2015, 13, 4197-4216. [CrossRef] 
78. Van Tran, T.T.; Truong, H.B.; Ha, N.; Tran, V.; Thu, T.M. Structure, conformation in aqueous solution and antimicrobial activity of ulvan extracted from green seaweed Ulva reticulata. Nat. Prod. Res. 2017, 6419, 1-6. [CrossRef] [PubMed]

79. Zouaoui, B.; Raho, B. The Phenolic Contents and Antimicrobial Activities of Some Marine Algae from the Mediterranean Sea (Algeria) 1. Russ. J. Mar. Biol. 2017, 43, 491-495. [CrossRef]

80. Ismail, A.; Ktari, L.; Ben Redjem Romdhane, Y.; Aoun, B.; Sadok, S.; Boudabous, A.; El Bour, M. Antimicrobial Fatty Acids from Green Alga Ulva rigida (Chlorophyta). Biomed Res. Int. 2018, 2018. [CrossRef] [PubMed]

81. Rhimou, B.; Hassane, R.; José, M.; Nathalie, B. The antibacterial potential of the seaweeds (Rhodophyaceae) of the Strait of Gibraltar and the Mediterranean coast of Morocco. Afr. J. Biotechnol. 2010, 9, 6365-6372. [CrossRef]

82. Chnadra, S. Textbook of Microbiology E Immunology; Elsevier: Amsterdam, The Netherlands, 2012.

83. Igo, M.; Schaffner, D. Quantifying the Influence of Relative Humidity, Temperature, and Diluent on the Survival and Growth of Enterobacter aerogenes. J. Food Prot. 2019, 82, 2135-2147. [CrossRef] [PubMed]

84. Harada, T.; Yamane, R.; Dang, V.C.; Nguyen, D.P.; Nguyen, T.A.D.; Jinnai, M.; Yonogi, S.; Kawahara, R.; Kanki, M.; Kawai, T.; et al. Prevalence and Antimicrobial Susceptibility of Enterobacteriaceae Isolated from Retail Pepper in Vietnam. J. Food Prot. 2016, 80, 716-724. [CrossRef] [PubMed]

85. Warren, B.R.; Parish, M.E.; Schneider, K.R. Shigella as a Foodborne Pathogen and Current Methods for Detection in Food. Crit. Rev. Food Sci. Nutr. 2006, 46, 551-567. [CrossRef]

86. Taneja, N.; Mewara, A. Shigellosis: Epidemiology in India. Indian J. Med. Res. 2016, 143, 565. [CrossRef]

87. Alizadeh-Hesar, M.; Bakhshi, B.; Najar-Peerayeh, S. Clonal dissemination of a single Shigella sonnei strain among Iranian children during Fall 2012 in Tehran, I.R. Iran. Infect. Genet. Evol. 2015, 34, 260-266. [CrossRef]

88. El Shafay, S.M.; Ali, S.S.; El-Sheekh, M.M. Antimicrobial activity of some seaweeds species from Red sea, against multidrug resistant bacteria. Egypt. J. Aquat. Res. 2016, 42, 65-74. [CrossRef]

89. Różalski, A.; Torzewska, A.; Moryl, M.; Kwil, I.; Maszewska, A.; Ostrowska, K.; Drzewiecka, D.; Zabłotni, A.; Palusiak, A.; Siwińska, M.; et al. Proteus sp.-An opportunistic bacterial pathogen-Classification, swarming growth, clinical significance and virulence factors. Folia Biol. Oecol. 2012, 8, 1-17. [CrossRef]

90. Drzewiecka, D. Significance and Roles of Proteus spp. Bacteria in Natural Environments. Microb. Ecol. 2016, 72, 741-758. [CrossRef]

91. Gong, Z.; Shi, X.; Bai, F.; He, X.; Zhang, H.; Li, Y.; Wan, Y.; Lin, Y.; Qiu, Y.; Chen, Q.; et al. Characterization of a Novel Diarrheagenic Strain of Proteus mirabilis Associated With Food Poisoning in China. Front. Microbiol. 2019, 10. [CrossRef] [PubMed]

92. Lei, C.-W.; Zhang, A.-Y.; Liu, B.-H.; Wang, H.-N.; Guan, Z.-B.; Xu, C.-W.; Xia, Q.-Q.; Cheng, H.; Zhang, D.-D. Molecular Characteristics of Salmonella Genomic Island 1 in Proteus mirabilis Isolates from Poultry Farms in China. Antimicrob. Agents Chemother. 2014, 58, 7570-7572. [CrossRef]

93. Lei, C.-W.; Zhang, A.-Y.; Wang, H.-N.; Liu, B.-H.; Yang, L.-Q.; Yang, Y.-Q. Characterization of SXT/R391 Integrative and Conjugative Elements in Proteus mirabilis Isolates from Food-Producing Animals in China. Antimicrob. Agents Chemother. 2016, 60, 1935-1938. [CrossRef]

94. Oni, V.; Oni, A.; Esumeh, F. Prevalence of Bacteria food poison from vegetable salads. Internet J. Nnutr. Wellness. 2009, 10, 1-5.

95. Li, X.; Du, Y.; Du, P.; Dai, H.; Fang, Y.; Li, Z.; Lv, N.; Zhu, B.; Kan, B.; Wang, D. SXT/R391 integrative and conjugative elements in Proteus species reveal abundant genetic diversity and multidrug resistance. Sci. Rep. 2016, 6, 37372. [CrossRef]

96. Anibijuwon, I.I.; Gbala, I.D.; Abioye, J.A.; Ogunlade, P.O. Antibiotic Sensitivity and Evaluation of Plasmid Profile of Major Foodborne Pathogens. J. Health Allied Sci. NU 2016, 6, 4-9. [CrossRef]

97. Al-Enazi, N.M.; Awaad, A.S.; Alqasoumi, S.I.; Alwethairi, M.F. Biological Activities of the Red Algae Galaxaura Rugosa and Liagora Hawaiiana Butters. Saudi Pharm. J. 2018, 26, 25-32. [CrossRef]

98. Moorthi, P.V. Antimicrobial properties of marine seaweed, Sargassum muticum against human pathogens. J. Coast. Life Med. 2015. [CrossRef]

99. Boonchum, W.; Peerapornpisal, Y.; Kanjanapothi, D.; Pekkoh, J.; Amornlerdpison, D.; Pumas, C.; Sangpaiboon, P.; Vacharapiyasophon, P. Antimicrobial and anti-inflammatory properties of various seaweeds from the Gulf of Thailand. Int. J. Agric. Biol. 2011, 13, 100-104.

100. Maheswari, M.U.; Reena, A.; Sivaraj, C. GC-MS analysis, antioxidant and antibacterial activity of the brown algae, Padina tetrastromatica. Int. J. Pharm. Sci. Res. 2017, 41, 84-93. [CrossRef] 
101. Manivannan, K.; Karthikai, G.; Anantharaman, P.; Balasubramanian, T. Antimicrobial potential of selected brown seaweeds from Vedalai coastal waters, Gulf of Mannar. Asian Pac. J. Trop. Biomed. 2011, 1, 114-120. [CrossRef]

102. Lopes, G.; Sousa, C.; Silva, L.R.; Pinto, E.; Andrade, P.B.; Bernardo, J.; Mouga, T.; Valentão, P. Can phlorotannins purified extracts constitute a novel pharmacological alternative for microbial infections with associated inflammatory conditions? PLoS ONE 2012, 7. [CrossRef] [PubMed]

103. Lines, C.C. Anti-Proliferative Activity of Meroditerpenoids Isolated from the Brown Alga Stypopodium flabelliforme against Several cancer cell lines. Mar. Drugs 2011, 852-862. [CrossRef]

104. Richardson, H.; Smaill, F. Recent advances: Medical microbiology. BMJ 1998, 317, 1060-1062. [CrossRef]

105. Lund, B.M. Provision of microbiologically safe food for vulnerable people in hospitals, care homes and in the community. Food Control 2019, 96, 535-547. [CrossRef]

106. Yang, X.; Wu, Q.; Zhang, J.; Huang, J.; Chen, L.; Wu, S.; Zeng, H.; Wang, J.; Chen, M.; Wu, H.; et al. Prevalence, Bacterial Load, and Antimicrobial Resistance of Salmonella Serovars Isolated From Retail Meat and Meat Products in China. Front. Microbiol. 2019, 10. [CrossRef]

107. Plumb, I.D.; Schwensohn, C.A.; Gieraltowski, L.; Tecle, S.; Schneider, Z.D.; Freiman, J.; Cote, A.; Noveroske, D.; Kolsin, J.; Brandenburg, J.; et al. Outbreak of Salmonella Newport Infections with Decreased Susceptibility to Azithromycin Linked to Beef Obtained in the United States and Soft Cheese Obtained in Mexico-United States, 2018-2019. MMWR Morb. Mortal. Wkly. Rep. 2019, 68, 713-717. [CrossRef]

108. Asfaw Ali, D.; Tadesse, B.; Ebabu, A. Prevalence and Antibiotic Resistance Pattern of Salmonella Isolated from Caecal Contents of Exotic Chicken in Debre Zeit and Modjo, Ethiopia. Int. J. Microbiol. 2020, 2020, 1-6. [CrossRef]

109. Kang, O.; Brice, O.; Lee, Y.; Chae, H. Antibacterial Activity of Ecklonia cava Against. Foodborne Pathog. Dis. 2010, 7, 435-441. [CrossRef]

110. Stoler, J.; Ahmed, H.; Asantewa Frimpong, L.; Bello, M. Presence of Pseudomonas aeruginosa in coliform-free sachet drinking water in Ghana. Food Control 2015, 55, 242-247. [CrossRef]

111. CDC. Antibiotic Resistance Threats in the United States; U.S. Department of Health and Human Services: Atlanta, GA, USA, 2019; pp. 1-113. [CrossRef]

112. Oludairo, O.O.; Aiyedun, J.O. Contamination of Commercially Packaged Sachet Water and the Public. Bangl. J. Vet. Med 2015, 13, 73-81. [CrossRef]

113. Banu, N.; Menakuru, H. Enumeration of microbial contaminants in sachet water: A public health challenge. Health (Irvine. Calif). 2010, 2, 582-588. [CrossRef]

114. Study, A.C.; Ibemesim, B.; Obinna, A. Comparative Study of Qualities of Sachet and Bottle Water Sold on the Streets of Abuja, Nigeria. S. Am. J. Public Health 2014, 2, 308-328.

115. Mgbakor, C.; Ojiegbe, G.C.; Okonko, I.O.; Odu, N.N.; Alli, J.A.; Nwanze, J.C.; Onoh, C.C. Bacteriological evaluation of some sachet water on sales in Owerri metropolis, Imo State, Nigeria. Malays. J. Microbiol. 2011, 7, 217-225. [CrossRef]

116. Brown, N.M.; Arbon, J.; Redpath, C. Contamination of milk-bank samples with Pseudomonas aeruginosa during pasteurization by penetration of organisms through the screw lid during cooling. J. Hosp. Infect. 2000, 46, 321-322. [CrossRef]

117. Maggs, C.A.; Gilmore, B.F. Antibiofilm Activity of the Brown Alga Halidrys siliquosa against Clinically Relevant Human Pathogens. Mar. Drugs 2015, 13, 3581-3605. [CrossRef]

118. Moubayed, N.M.S.; Al Houri, H.J.; Al Khulaifi, M.M.; Al Farraj, D.A. Antimicrobial, antioxidant properties and chemical composition of seaweeds collected from Saudi Arabia (Red Sea and Arabian Gulf). Saudi J. Biol. Sci. 2017, 24, 162-169. [CrossRef]

119. Sasidharan, S.; Darah, I.; Noordin, M.K.M.J. In Vitro antimicrobial activity against Pseudomonas aeruginosa and acute oral toxicity of marine algae Gracilaria changii. New Biotechnol. 2010, 27, 390-396. [CrossRef]

120. Stabili, L.; Acquaviva, M.I.; Biandolino, F.; Cavallo, R.A.; De Pascali, S.A.; Fanizzi, F.P.; Narracci, M.; Cecere, E.; Petrocelli, A. Biotechnological potential of the seaweed Cladophora rupestris (Chlorophyta, Cladophorales) lipidic extract. New Biotechnol. 2014, 31, 436-444. [CrossRef] [PubMed]

121. Lee, J.-H.; Eom, S.-H.; Lee, E.-H.; Jung, Y.-J.; Kim, H.-J.; Jo, M.-R.; Son, K.-T.; Lee, H.-J.; Kim, J.H.; Lee, M.-S.; et al. In Vitro antibacterial and synergistic effect of phlorotannins isolated from edible brown seaweed Eisenia bicyclis against acne-related bacteria. Algae 2014, 29, 47-55. [CrossRef] 
122. Loir, Y.L.; Baron, F.; Gautier, M. Staphylococcus aureus and food poisoning. Genet. Mol. Res. 2003, 7-28. [CrossRef]

123. Atanassova, V.; Meindl, A.; Ring, C. Prevalence of Staphylococcus aureus and staphylococcal enterotoxins in raw pork and uncooked smoked ham-A comparison of classical culturing detection and RFLP-PCR. Int. J. Food Microbiol. 2001, 68, 105-113. [CrossRef]

124. Fisher, E.L.; Otto, M.; Cheung, G.Y.C. Basis of virulence in enterotoxin-mediated staphylococcal food poisoning. Front. Microbiol. 2018, 9, 1-18. [CrossRef]

125. Gutiérrez-Rodríguez, A.G.; Juárez-Portilla, C.; Olivares-Bañuelos, T.; Zepeda, R.C. Anticancer activity of seaweeds. Drug Discov. Today. 2018, 23, 434-447. [CrossRef]

126. Benassai, E.; Del Bubba, M.; Ancillotti, C.; Colzi, I.; Gonnelli, C.; Calisi, N.; Salvatici, M.C.; Casalone, E.; Ristori, S. Green and cost-effective synthesis of cooper nanoparticles by extract of non-edible and waste plant material from Vaccinium species: Characterization and antimicrobial activity. Mater. Aci. Eng. C 2021, 119, 111453. [CrossRef]

127. Águila-Ramírez, R.N.; Arenas-González, A.; Hernández-Guerrero, C.J.; González-Acosta, B.; Borges-Souza, J.M.; Véron, B.; Pope, J.; Hellio, C. Antimicrobial and antifouling activities achieved by extracts of seaweeds from Gulf of California, Mexico. Hidrobiologica 2012, 22, 8-15.

128. Massironi, A.; Morelli, A.; Grassi, L.; Puppi, D.; Braccini, S.; Maisetta, G.; Esin, S.; Batoni, G.; Della Pinta, C.; Chiellini, F. Ulvan as novel reducing and stabilizing agent from renewable algal biomass: Application to green synthesis of sillver nanoparticles. Carbohydr. Polym. 2019, 203, 310-321. [CrossRef]

129. Tajbakhsh, S.; Pouyan, M.; Zandi, K.; Bahramian, P.; Sartavi, K.; Fouladvand, M.; Asayesh, G.; Barazesh, A. In Vitro study of antibacterial activity of the alga Sargassum oligocystum from the Persian Gulf. Eur. Rev. Med. Pharmacol. Sci. 2011, 15, 293-298.

130. Ghania, A.; Nabila, B.B.; Larbi, B.; Elisabeth, M.; Philippe, G.; Mariem, B.; Khadidja, K.K.; Wacila, B.R.; Fawzia, A.B. Antimicrobial and antiparasitic activities of three algae from the northwest coast of Algeria. Nat. Prod. Res. 2019, 33, 742-745. [CrossRef] [PubMed]

131. Hoare, A.H.; Tan, S.P.; Mcloughlin, P.; Mulhare, P.; Hughes, H. The Screening and Evaluation of Fucus serratus and Fucus vesiculosus Extracts against Current Strains of MRSA Isolated from a Clinical Hospital Setting. Sci. Rep. 2019, 1-9. [CrossRef]

132. Baliano, A.P.; Pimentel, E.F.; Buzin, A.R.; Vieira, T.Z.; Romão, W.; Tose, L.V.; Lenz, D.; de Andrade, T.U.; Fronza, M.; Kondratyuk, T.P.; et al. Brown seaweed Padina gymnospora is a prominent natural wound-care product. Braz. J. Pharmacogn. 2016, 26, 714-719. [CrossRef]

133. Iannetti, L.; Schirone, M.; Neri, D.; Visciano, P.; Acciari, V.A.; Centorotola, G.; Mangieri, M.S.; Torresi, M.; Santarelli, G.A.; Di Marzio, V.; et al. Listeria monocytogenes in poultry: Detection and strain characterization along an integrated production chain in Italy. Food Microbiol. 2020, 91, 103533. [CrossRef] [PubMed]

134. McLauchlin, J.; Mitchell, R.T.; Smerdon, W.J.; Jewell, K. Listeria monocytogenes and listeriosis: A review of hazard characterisation for use in microbiological risk assessment of foods. Int. J. Food Microbiol. 2004, 92, 15-33. [CrossRef]

135. Szymczak, B.; Szymczak, M.; Trafiałek, J. Prevalence of Listeria species and L. monocytogenes in ready-to-eat foods in the West Pomeranian region of Poland: Correlations between the contamination level, serogroups, ingredients, and producers. Food Microbiol. 2020, 91, 103532. [CrossRef]

136. Jackson, K.A.; Gould, L.H.; Hunter, J.C.; Kucerova, Z.; Jackson, B. Listeriosis Outbreaks Associated with Soft Cheeses, United States, 1998-20141. Emerg. Infect. Dis. 2018, 24, 1116-1118. [CrossRef]

137. Cox, S. An Assessment of the Antioxidant and Antimicrobial Activity of Six Species of Edible Irish Seaweeds. Int. Food Res. J. 2010. [CrossRef]

138. Terra, M.R.; Costa, L.C.; Dardaque, R.M.; Furlaneto, M.C.; Furlaneto-maia, L. Food as a potencial reservoir of enterococcus that host dererminants of virulence and resistence. Braz. J. Surg. Clin. Res. BJSCR 2018, 22, $86-93$.

139. Nami, Y.; Bakhshayesh, R.V.; Jalaly, H.M.; Lotfi, H.; Eslami, S.; Hejazi, M.A. Probiotic properties of enterococcus isolated from artisanal dairy products. Front. Microbiol. 2019, 10, 1-13. [CrossRef]

140. Murray, B.E. The life and times of the enterococcus. Clin. Microbiol. Rev. 1990, 3, 46-65. [CrossRef]

141. Giraffa, G. Enterococci from foods. FEMS Microbiol. Rev. 2002, 26, 163-171. [CrossRef] 
142. Ismail, A.; Ktari, L.; Ahmed, M.; Bolhuis, H.; Bouhaouala-Zahar, B.; Stal, L.J.; Boudabbous, A.; El Bour, M. Heterotrophic bacteria associated with the green alga Ulva rigida: Identification and antimicrobial potential. J. Appl. Phycol. 2018, 30, 2883-2899. [CrossRef]

143. Chorin, E.; Thuault, D.; Cléret, J.-J.; Bourgeois, C.-M. Modelling Bacillus cereus growth. Int. J. Food Microbiol. 1997, 38, 229-234. [CrossRef]

144. Stenfors Arnesen, L.P.; Fagerlund, A.; Granum, P.E. From soil to gut: Bacillus cereus and its food poisoning toxins. FEMS Microbiol. Rev. 2008, 32, 579-606. [CrossRef] [PubMed]

145. Huang, Y.; Flint, S.H.; Palmer, J.S. Bacillus cereus spores and toxins-The potential role of biofilms. Food Microbiol. 2020, 90, 103493. [CrossRef]

146. Zhao, S.; Chen, J.; Fei, P.; Feng, H.; Wang, Y.; Ali, M.A.; Li, S.; Jing, H.; Yang, W. Prevalence, molecular characterization, and antibiotic susceptibility of Bacillus cereus isolated from dairy products in China. J. Dairy Sci. 2020, 103, 3994-4001. [CrossRef]

147. Yu, P.; Yu, S.; Wang, J.; Guo, H.; Zhang, Y.; Liao, X.; Zhang, J.; Wu, S.; Gu, Q.; Xue, L.; et al. Bacillus cereusIsolated from vegetables in China: Incidence, genetic diversity, virulence genes, and antimicrobial resistance. Front. Microbiol. 2019, 10, 1-10. [CrossRef]

148. Patra, J.K.; Baek, K.H. Antibacterial activity and action mechanism of the essential oil from enteromorpha linza L. against foodborne pathogenic bacteria. Molecules 2016, 21, 388. [CrossRef] [PubMed]

149. Alves-Barroco, C.; Roma-Rodrigues, C.; Raposo, L.R.; Brás, C.; Diniz, M.; Caço, J.; Costa, P.M.; Santos-Sanches, I.; Fernandes, A.R. Streptococcus dysgalactiae subsp. dysgalactiae isolated from milk of the bovine udder as emerging pathogens: In Vitro and in vivo infection of human cells and zebrafish as biological models. Microbiologyopen 2019, 8, e00623. [CrossRef] [PubMed]

150. Yamaguchi, T.; Kawahara, R.; Katsukawa, C.; Kanki, M.; Harada, T.; Yonogi, S.; Iwasaki, S.; Uehara, H.; Okajima, S.; Nishimura, H.; et al. Foodborne Outbreak of Group G Streptococcal Pharyngitis in a School Dormitory in Osaka, Japan. J. Clin. Microbiol. 2018, 56. [CrossRef] [PubMed]

151. Mesquita, A.A.; Rocha, C.M.B.M.; Bruhn, F.R.P.; Custódio, D.A.C.; Braz, M.S.; Pinto, S.M.; Silva, D.B.; Costa, G.M. Staphylococcus aureus and Streptococcus agalactiae: Prevalence, resistance to antimicrobials, and their relationship with the milk quality of dairy cattle herds in Minas Gerais state, Brazil. Pesqui. Vet. Bras. 2019, 39, 308-316. [CrossRef]

152. Riekerink, R.G.M.O.; Barkema, H.W.; Veenstra, S.; Poole, D.E.; Dingwell, R.T.; Keefe, G.P. Prevalence of contagious mastitis pathogens in bulk tank milk in Prince Edward Island. Can. Vet. J. 2006, 46, 567-572.

153. Jans, C.; Kaindi, D.W.M.; Böck, D.; Njage, P.M.K.; Kouamé-Sina, S.M.; Bonfoh, B.; Lacroix, C.; Meile, L. Prevalence and comparison of Streptococcus infantarius subsp. infantarius and Streptococcus gallolyticus subsp. macedonicus in raw and fermented dairy products from East and West Africa. Int. J. Food Microbiol. 2013, 167, 186-195. [CrossRef]

154. Manilal, A.; Sujith, S.; Kiran, G.S.; Selvin, J.; Shakir, C.; Gandhimathi, R.; Lipton, A.P. Antimicrobial potential and seasonality of red algae collected from the southwest coast of India tested against shrimp, human and phytopathogens. Ann. Microbiol. 2009, 59, 207-219. [CrossRef]

155. Stabili, L.; Acquaviva, M.I.; Biandolino, F.; Cavallo, R.A.; De Pascali, S.A.; Fanizzi, F.P.; Narracci, M.; Petrocelli, A.; Cecere, E. The lipidic extract of the seaweed Gracilariopsis longissima (Rhodophyta, Gracilariales): A potential resource for biotechnological purposes? New Biotechnol. 2012, 29, 443-450. [CrossRef] [PubMed]

156. Berri, M.; Slugocki, C.; Olivier, M.; Helloin, E.; Jacques, I.; Salmon, H.; Demais, H.; Le Goff, M.; Collen, P.N. Marine-sulfated polysaccharides extract of Ulva armoricana green algae exhibits an antimicrobial activity and stimulates cytokine expression by intestinal epithelial cells. J. Appl. Phycol. 2016, 28, 2999-3008. [CrossRef]

157. Osman, M.E.H.; Abushady, A.M.; Elshobary, M.E. In Vitro screening of antimicrobial activity of extracts of some macroalgae collected from Abu-Qir bay Alexandria, Egypt. Afr. J. Biotechnol. 2010, 9, 7203-7208. [CrossRef]

158. Carocho, M.; Morales, P.; Ferreira, I.C.F.R. Antioxidants: Reviewing the chemistry, food applications, legislation and role as preservatives. Trends Food Sci. Technol. 2018, 71, 107-120. [CrossRef]

159. Carocho, M.; Barreiro, M.F.; Morales, P.; Ferreira, I.C.F.R. Adding Molecules to Food, Pros and Cons: A Review on Synthetic and Natural Food Additives. Compr. Rev. Food Sci. Food Saf. 2014, 13, 377-399. [CrossRef] 
160. Safafar, H.; van Wagenen, J.; Møller, P.; Jacobsen, C. Carotenoids, Phenolic Compounds and Tocopherols Contribute to the Antioxidative Properties of Some Microalgae Species Grown on Industrial Wastewater. Mar. Drugs 2015, 13, 7339-7356. [CrossRef] [PubMed]

161. Jönsson, M.; Allahgholi, L.; Sardari, R.R.R.; Hreggviosson, G.O.; Karlsson, E.N. Extraction and modification of macroalgal polysaccharides for current and next-generation applications. Molecules 2020, 25, 930. [CrossRef]

162. Vuong, D.; Kaplan, M.; Lacey, H.J.; Crombie, A.; Lacey, E.; Piggott, A.M. A study of the chemical diversity of macroalgae from South Eastern Australia. Fitoterapia 2018, 126, 53-64. [CrossRef] [PubMed]

163. MacArtain, P.; Gill, C.I.R.; Brooks, M.; Campbell, R.; Rowland, I.R. Nutritional Value of Edible Seaweeds. Nutr. Rev. 2008, 65, 535-543. [CrossRef]

164. Li, Y.; Yang, Z.; Li, J. Shelf-life extension of Pacific white shrimp using algae extracts during refrigerated storage. J. Sci. Food Agric. 2017, 97, 291-298. [CrossRef] [PubMed]

165. Arulkumar, A.; Paramasivam, S.; Miranda, J.M. Combined Effect of Icing Medium and Red Alga Gracilaria verrucosa on Shelf Life Extension of Indian Mackerel (Rastrelliger kanagurta). Food Bioprocess Technol. 2018, 11, 1911-1922. [CrossRef]

166. Oucif, H.; Miranda, J.M.; Mehidi, S.A.; Abi-Ayad, S.-M.E.-A.; Barros-Velázquez, J.; Aubourg, S.P. Effectiveness of a combined ethanol-aqueous extract of alga Cystoseira compressa for the quality enhancement of a chilled fatty fish species. Eur. Food Res. Technol. 2018, 244, 291-299. [CrossRef]

167. Barbosa, R.G.; Trigo, M.; Campos, C.A.; Aubourg, S.P. Preservative Effect of Algae Extracts on Lipid Composition and Rancidity Development in Brine-Canned Atlantic Chub Mackerel (Scomber colias). Eur. J. Lipid Sci. Technol. 2019, 121, 1900129. [CrossRef]

168. Blanco-Pascual, N.; Gómez-Guillén, M.C.; Montero, M.P. Integral Mastocarpus stellatus use for antioxidant edible film development. Food Hydrocoll. 2014, 40, 128-137. [CrossRef]

169. Alemán, A.; Blanco-Pascual, N.; Montero, M.P.; Gómez-Guillén, M.C. Simple and efficient hydrolysis procedure for full utilization of the seaweed Mastocarpus stellatus to produce antioxidant films. Food Hydrocoll. 2016, 56, 277-284. [CrossRef]

170. García-Soto, B.; Miranda, J.M.; Rodríguez-Bernaldo de Quirós, A.; Sendón, R.; Rodríguez-Martínez, A.V.; Barros-Velázquez, J.; Aubourg, S.P. Effect of biodegradable film (lyophilised alga Fucus spiralis and sorbic acid) on quality properties of refrigerated megrim (Lepidorhombus whiffiagonis). Int. J. Food Sci. Technol. 2015, 50, 1891-1900. [CrossRef]

171. Rodríguez-Martínez, A.V.; Sendón, R.; Abad, M.J.; González-Rodríguez, M.V.; Barros-Velázquez, J.; Aubourg, S.P.; Paseiro-Losada, P.; Rodríguez-Bernaldo de Quirós, A. Migration kinetics of sorbic acid from polylactic acid and seaweed based films into food simulants. LWT Food Sci. Technol. 2016, 65, 630-636. [CrossRef]

172. Gomaa, M.; Fawzy, M.A.; Hifney, A.F.; Abdel-Gawad, K.M. Use of the brown seaweed Sargassum latifolium in the design of alginate-fucoidan based films with natural antioxidant properties and kinetic modeling of moisture sorption and polyphenolic release. Food Hydrocoll. 2018, 82, 64-72. [CrossRef]

173. Gomaa, M.; Hifney, A.F.; Fawzy, M.A.; Abdel-Gawad, K.M. Use of seaweed and filamentous fungus derived polysaccharides in the development of alginate-chitosan edible films containing fucoidan: Study of moisture sorption, polyphenol release and antioxidant properties. Food Hydrocoll. 2018, 82, 239-247. [CrossRef]

174. Doh, H.; Dunno, K.D.; Whiteside, W.S. Preparation of novel seaweed nanocomposite film from brown seaweeds Laminaria japonica and Sargassum natans. Food Hydrocoll. 2020, 105, 105744. [CrossRef]

175. He, Q.; Huang, Y.; Lin, B.; Wang, S. A nanocomposite film fabricated with simultaneously extracted protein-polysaccharide from a marine alga and $\mathrm{TiO}_{2}$ nanoparticles. J. Appl. Phycol. 2017, 29, 1541-1552. [CrossRef]

176. Augusto, A.; Dias, J.R.; Campos, M.J.; Alves, N.M.; Pedrosa, R.; Silva, S.F.J. Influence of Codium tomentosum Extract in the Properties of Alginate and Chitosan Edible Films. Foods 2018, 7, 53. [CrossRef] [PubMed]

177. Bi, F.; Zhang, X.; Liu, J.; Yong, H.; Gao, L.; Liu, J. Development of antioxidant and antimicrobial packaging films based on chitosan, D- $\alpha$-tocopheryl polyethylene glycol 1000 succinate and silicon dioxide nanoparticles. Food Packag. Shelf Life 2020, 24, 100503. [CrossRef]

178. Junter, G.-A.; Thébault, P.; Lebrun, L. Polysaccharide-based antibiofilm surfaces. Acta Biomater. 2016, 30, 13-25. [CrossRef] [PubMed]

179. Yılmaz Öztürk, B.; Yenice Gürsu, B.; Dağ, İ. Antibiofilm and antimicrobial activities of green synthesized silver nanoparticles using marine red algae Gelidium corneum. Process Biochem. 2020, 89, 208-219. [CrossRef] 
180. Dahms, H.U.; Dobretsov, S. Antifouling Compounds from Marine Macroalgae. Mar. Drugs 2017, 15, 265. [CrossRef]

181. King, T.; Cole, M.; Farber, J.M.; Eisenbrand, G.; Zabaras, D.; Fox, E.M.; Hill, J.P. Food safety for food security: Relationship between global megatrends and developments in food safety. Trends Food Sci. Technol. 2017, 68, 160-175. [CrossRef]

182. Rai, M.; Chikindas, M. Natural Antimicrobials in Food Safety and Quality; CABI: Wallingford, UK, 2011; ISBN 9781845937690.

183. Aloui, H.; Khwaldia, K. Natural Antimicrobial Edible Coatings for Microbial Safety and Food Quality Enhancement. Compr. Rev. Food Sci. Food Saf. 2016, 15, 1080-1103. [CrossRef]

184. Arshad, M.S.; Batool, S.A. Natural Antimicrobials, their Sources and Food Safety. Food Addit. 2017. [CrossRef]

185. Fu, Y.; Sarkar, P.; Bhunia, A.K.; Yao, Y. Delivery systems of antimicrobial compounds to food. Trends Food Sci. Technol. 2016, 57, 165-177. [CrossRef]

186. Gupta, S.; Abu-Ghannam, N. Recent developments in the application of seaweeds or seaweed extracts as a means for enhancing the safety and quality attributes of foods. Innov. Food Sci. Emerg. Technol. 2011, 12, 600-609. [CrossRef]

187. Pisoschi, A.M.; Pop, A.; Georgescu, C.; Turcuş, V.; Olah, N.K.; Mathe, E. An overview of natural antimicrobials role in food. Eur. J. Med. Chem. 2018, 143, 922-935. [CrossRef]

188. Abu-Ghannam, N.; Rajauria, G. Antimicrobial activity of compounds isolated from algae. In Functional Ingredients from Algae for Foods and Nutraceuticals; Woodhead Publishing Limited: Sawston, UK, 2013; pp. 287-306, ISBN 9780857095121.

189. Triandafyllidou, A.; McAuliffe, M. Report overview. Migr. Smuggling Data Res. 2019, 1-18. [CrossRef]

190. Gillis, R.; Adams, G.; Besong, D.; Machova, E.; Ebringerova, A.; Harding, S.; Patel, T. Application of novel analytical ultracentrifuge analysis to solutions of fungal mannans. Eur. Biophys. J. 2017, 46, 235-245. [CrossRef]

191. Wells, M.L.; Potin, P.; Craigie, J.S.; Raven, J.A.; Merchant, S.S.; Helliwell, K.E.; Smith, A.G.; Camire, M.E.; Brawley, S.H. Algae as nutritional and functional food sources: Revisiting our understanding. J. Appl. Phycol. 2017, 29, 949-982. [CrossRef]

192. Caporgno, M.P.; Mathys, A. Trends in Microalgae Incorporation Into Innovative Food Products With Potential Health Benefits. Front. Nutr. 2018, 5. [CrossRef]

193. Neetoo, H.; Ye, M.; Chen, H. Bioactive alginate coatings to control Listeria monocytogenes on cold-smoked salmon slices and fillets. Int. J. Food Microbiol. 2010, 136, 326-331. [CrossRef]

Publisher's Note: MDPI stays neutral with regard to jurisdictional claims in published maps and institutional affiliations.

(C) 2020 by the authors. Licensee MDPI, Basel, Switzerland. This article is an open access article distributed under the terms and conditions of the Creative Commons Attribution (CC BY) license (http://creativecommons.org/licenses/by/4.0/). 